\title{
DONG PINGPING
}

\section{Minoration de combinaisons linéaires de deux logarithmes $p$-adiques}

\author{
Annales de la faculté des sciences de Toulouse $5^{e}$ série, tome 12, \\ $\mathrm{n}^{\circ} 2$ (1991), p. 195-250 \\ <http://www.numdam.org/item?id=AFST_1991_5_12_2_195_0>
}

(C) Université Paul Sabatier, 1991, tous droits réservés.

L'accès aux archives de la revue «Annales de la faculté des sciences de Toulouse » (http://picard.ups-tlse.fr/ annales/) implique l'accord avec les conditions générales d'utilisation (http://www.numdam.org/conditions). Toute utilisation commerciale ou impression systématique est constitutive d'une infraction pénale. Toute copie ou impression de ce fichier doit contenir la présente mention de copyright.

\section{Numdam}

Article numérisé dans le cadre du programme Numérisation de documents anciens mathématiques http://www.numdam.org/ 


\title{
Minoration de combinaisons linéaires de deux logarithmes $p$-adiques
}

\author{
Dong PINGPING ${ }^{(*)}$
}

\begin{abstract}
RÉSUMÉ. - En utilisant la méthode de Schneider dans un domaine ultramétrique, nous donnons une minoration explicite d'une combinaison linéaire de deux logarithmes $p$-adiques de nombres algébriques à coefficients rationnels. C'est la première version quantitative d'un travail de Veldkamp en 1936. Des minorations explicites semblables aux nôtres (mais plus faibles) avaient déjà été obtenues en 1967 par Schinzel qui utilisait la méthode de Gel'fond. Nous comparons aussi notre résultat à celui de Yu Kunrui qui repose sur la méthode de Baker. Ce travail constitue une traduction p-adique de minorations récemment obtenues par Mignotte et Waldschmidt.
\end{abstract}

Abstract. - Using Schneider's method in an ultrametric field, we give an explicit lower bound for a linear form in two p-adic logarithms of algebraic numbers with rational coefficients. This is the first quantitative version of Veldkamp's work in 1936. Explicit estimates had been derived by Schinzel in 1967 using Gel'fond's method, but his estimates are weaker than our. We also compare our result with Yu Kunrui's one. Our work is a p-adic translation of recent results by Mignotte and Waldschmidt.

\section{Introduction}

En 1935, Mahler [2] a établi que si $p$ est un nombre premier, et $\alpha_{1}, \alpha_{2}$ sont deux nombres algébriques différents de 1 vérifiant $\left|\alpha_{1}-1\right|_{p} \leq 1 / p,\left|\alpha_{2}-1\right|_{p} \leq$ $1 / p$, alors le quotient des deux logarithmes $p$-adiques $\log \alpha_{1} / \log \alpha_{2}$ est soit rationnel, soit transcendant. La démonstration de Mahler utilisait la méthode qui avait permis à Gel'fond, l'année précédente, de résoudre le septième problème de Hilbert sur la transcendance des nombres complexes

(*) "Problèmes Diophantiens", CNRS et Univ. P. et M. Curie, 11, rue P. et M. Curie, 75231 Paris Cedex 05, France. 
$\log \alpha_{1} / \log \alpha_{2}$. En 1936, Veldkamp donne une deuxième démonstration de ce résultat de Malher grâce à la méthode introduite par Schneider pour résoudre ce septième problème de Hilbert.

Dès 1935 , Gel'fond avait obtenu une minoration explicite pour des combinaisons linéaires de deux logarithmes (complexes) de nombres algébriques. En 1939, il raffine ce résultat et en démontre aussi l'analogue $p$-adique. Ces deux énoncés (complexe et $p$-adique) ont été entièrement explicités par Schinzel [6] en 1967 qui en donne diverses applications arithmétiques. La méthode est celle de Gel'fond (et Mahler pour le cas ultramétrique). C'est seulement en 1978 que la méthode de Schneider a été utilisée pour minorer effectivement (et même explicitement) les combinaisons linéaires de deux logarithmes. Les trois articles [3] de Mignotte et Waldschmidt concernent seulement le cas complexe, et nous allons ici en donner la première version ultramétrique.

Notre théorème principal est énoncé au paragraphe 4 . En voici un cas particulier. On désigne par $\alpha_{1}, \alpha_{2}$ deux nombres algébriques non nuls, par $p$ un nombre premier, et par $b_{1}, b_{2}$ deux nombres entiers. On suppose

$$
\nu\left(\alpha_{i}-1\right)>\frac{1}{p-1}, \quad(i=1,2),
$$

où $\nu$ désigne un prolongement à une extension finie $\mathbb{K} \operatorname{de} \mathbb{Q}$, avec $\alpha_{1}, \alpha_{2} \in \mathbb{K}$, de la valuation $p$-adique sur $\mathbb{Q}$.

On note ensuite

$$
a_{i}=\max \left\{h\left(\alpha_{i}\right), 2 \log p\right\}, \quad(i=1,2),
$$

où $h$ est la hauteur logarithmique absolue, et

$$
B=\max \left\{\left\|b_{1}\right\|,\left\|b_{2}\right\|, 3\right\},
$$

où $\|\cdot\|$ désigne la valeur absolue usuelle sur $\mathbb{Q}$.

Enfin, $D$ désigne le degré de $\mathbb{K}$ sur $\mathbb{Q}$.

Corollaire 1.1. - Si $\alpha_{1}^{b_{1}} \neq \alpha_{2}^{b_{2}}$, alors

$$
\nu\left(\alpha_{1}^{b_{1}}-\alpha_{2}^{b_{2}}\right) \leq \frac{(51 p+67)^{2}}{(\log p)^{4}} D^{4} a_{1} a_{2}(\log B)^{2} .
$$

Notre énoncé concerne seulement les combinaisons linéaires de deux logarithmes $p$-adiques (c'est-à-dire des majorations de $\nu\left(\alpha_{1}^{b_{1}} \alpha_{2}^{b_{2}}-1\right)$ ). On 
connaît des estimations valables pour un nombre quelconque de logarithmes, à commencer par les résultats non effectifs de Gel'fond en 1949 (reposant sur les théorèmes d'approximation de Thue, Siegel, Roth et leurs analogues non archimédiens), sans oublier ceux que l'on obtient par la méthode de Baker (voir [4], [5] et [10]). Les estimations explicites les plus précises actuellement connues étaient celles de Yu Kunrui [10]. Quand le paramètre $B$ n'est pas trop grand, notre corollaire 1.1 est souvent plus précis que celui de [10]. Dans des applications numériques, par exemple pour résoudre complètement certaines équations diophantiennes, le fait que nos valeurs numériques soient petites est très utile.

Enfin, nous renvoyons à l'article [5] et à sa bibliographie pour des références plus complètes aux travaux de Veldkamp, Gel'fond, Günther, Içen, Brumer, Coates, Sprindzuk, Kaufman, Loxton et van der Poorten.

Nous fixons d'abord les notations, puis nous comparons notre corollaire 1.1 aux résultats de Yu Kunrui. Le plan de cet article est le suivant. Dans le deuxième paragraphe nous énonçons quelques lemmes classiques que nous utiliserons plus tard. Le troisième paragraphe est consacré à la démonstration d'une estimation $p$-adique pour un produit de nombres rationnels; c'est l'analogue ultramétrique de résultats dans la section 3 de [3] II et III. Le théorème principal de cet article est énoncé et démontré au paragraphe 4.

Nous utiliserons les notations suivantes :

- $\mathbb{K}$ une extention finie de $\mathbb{Q}$;

- $D=[\mathbb{K}: \mathbb{Q}]$;

$\bullet \mathfrak{p}$ un idéal premier de $\mathbb{K}$;

- $p$ la caractéristique résiduelle de $\mathfrak{p}$;

- $f$ l'entier positif vérifiant $N(\mathfrak{p})=p^{f}$

- e l'indice de ramification de $\mathfrak{p}: \mathfrak{p}^{\mathrm{e}} \| p$

- | | la valeur absolue $\mathfrak{p}$-adique sur $\mathbb{K}$, normalisée par

$$
|x|=p^{-\nu(x)}, \quad x \in \mathbb{K},
$$

où $\nu(x)=(1 / \mathrm{e}) \operatorname{ord}_{\mathfrak{p}} x, \operatorname{ord}_{\mathfrak{p}} x$ étant le plus grand entier $m$ tel que

$$
\begin{gathered}
\mathfrak{p}^{m} \mid x \quad\left(\operatorname{ord}_{\mathfrak{p}} 0=\infty\right) \\
\text { - } g_{\mathfrak{p}}=\left[\frac{1}{2}+\frac{\mathrm{e}}{p-1}\right], G_{\mathfrak{p}}=N\left(\mathfrak{p}^{g_{\mathfrak{p}}}\right)(N(\mathfrak{p})-1) \leq p^{D} ;
\end{gathered}
$$




\section{Dong Pingping}

- $\alpha_{1}, \alpha_{2}$ deux éléments de $\mathbb{K}$;

- $b_{1}, b_{2}$ deux entiers rationnels.

Écrivons $\Lambda=\alpha_{1}^{b_{1}}-\alpha_{2}^{b_{2}}$ et supposons désormais $\Lambda \neq 0$.

On énonce maintenant deux théorèmes de Yu Kunrui donnant des minorations $p$-adiques de $\Lambda$ dans son article [9], qui est publié avant son article [10]. Ces résultats imposent certaines restrictions sur les $\alpha_{i}$. Les énoncés de Yu Kunrui concernent plus généralement les nombres de la forme $\left|\alpha_{1}^{b_{1}} \cdots \alpha_{n}^{b_{n}}-1\right|_{p}$, mais nous nous restreignons ici au cas $n=2$.

On pose ici

$$
\begin{aligned}
& a_{i} \geq \max \left\{h\left(\alpha_{i}\right), \frac{f \log p}{D}\right\}, \quad i=1,2 \\
& B_{0} \geq \min _{b_{i} \neq 0}\left\|b_{i}\right\|, \quad B_{i} \geq\left\|b_{i}\right\|, \quad i=1,2 \\
& W_{1} \geq\left\{\begin{array}{l}
\max \left\{\log \left(1+\frac{3}{16} \frac{f \log p}{D}\left(\frac{B_{2}}{a_{1}}+\frac{B_{1}}{a_{2}}\right)\right), \log B_{0}, \frac{f \log p}{D}\right\} \\
\max \left\{\log \left(1+\frac{3}{16} \frac{f \log p}{D}\left(\frac{B_{2}}{a_{1}}+\frac{B_{1}}{a_{2}}\right)\right), \frac{f \log p}{D}\right\}_{\text {si } p \nmid\left(b_{1}, b_{2}\right)}
\end{array}\right. \\
& W_{2} \geq\left\{\begin{array}{l}
\max \left\{\log \left(1+\frac{1}{5} \frac{f \log p}{D}\left(\frac{B_{2}}{a_{1}}+\frac{B_{1}}{a_{2}}\right)\right), \log B_{0}, \frac{f \log p}{D}\right\} \\
\max \left\{\log \left(1+\frac{1}{5} \frac{f \log p}{D}\left(\frac{B_{2}}{a_{1}}+\frac{B_{1}}{a_{2}}\right)\right), \frac{f \log p}{D}\right\}_{\text {si } p \nmid\left(b_{1}, b_{2}\right)}
\end{array}\right.
\end{aligned}
$$

On suppose

$$
\begin{aligned}
& \nu\left(\alpha_{i}\right)=0, \quad i=1,2 \\
& \mathbb{K}=\mathbb{Q}\left(\alpha_{1}, \alpha_{2}\right)
\end{aligned}
$$

et $q$ est un nombre premier vérifiant

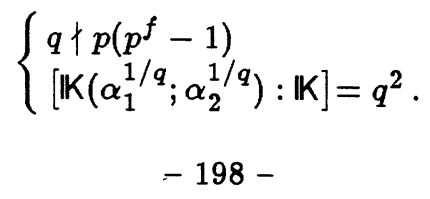


Le premier théorème :

$$
\begin{aligned}
\operatorname{ord}_{\mathfrak{p}} \Lambda \leq & C_{1}(p) \frac{50176}{225} \sqrt{2} \mathrm{e}^{2} q^{4}(q-1) \log ^{2}(2 q)\left(p^{f}-1\right) \frac{(2+1 /(p+1))^{2}}{(f \log p)^{4}} \times \\
& \times D^{4} a_{1} a_{2}\left(\frac{W_{1}}{12}+\log (4 D)\right)\left\{\log \left(4 D \max \left(1, a_{1}, a_{2}\right)\right)+\frac{f \log p}{16}\right\}
\end{aligned}
$$

où

$$
\begin{gathered}
C_{1}(2)=768523 \quad ; \quad C_{1}(3)=167881 \\
C_{1}(p)=87055\left(2+\frac{1}{p-1}\right)^{2}, \quad p \geq 5 .
\end{gathered}
$$

Le deuxième théorème :

$$
\begin{aligned}
\operatorname{ord}_{\mathfrak{p}} \Lambda \leq & C_{2}(p) \frac{2048 \mathrm{e}}{9} \sqrt{2} \mathrm{e}^{2} q^{4}(q-1) \log ^{2}(2 q)\left(p^{f}-1\right) \frac{(2+1 /(p-1))^{2}}{(f \log p)^{4}} \times \\
& \times D^{4} a_{1} a_{2}\left(\frac{W_{2}}{12}+\log (4 D)\right)^{2}
\end{aligned}
$$

où

$$
\begin{gathered}
C_{2}(2)=338071 ; \quad C_{2}(3)=61716 \\
C_{2}(p)=14491\left(2+\frac{1}{p-1}\right)^{3}, \quad p \geq 5
\end{gathered}
$$

Nous modifions ici le sens des quantités $a_{1}, a_{2}, B_{0}, B_{1}, B_{2}$ en prenant (2) et (3), et remplaçons la condition (6) par (1); nous aurons donc une minoration de $\log |\Lambda|$ (corol. 1.1) bien différent de celle de Yu Kunrui.

Dans les démonstrations des théorèmes de Yu Kunrui et du corollaire 1.1, les conditions sur les nombres algébriques $\alpha_{1}, \alpha_{2}$ sont toutes nécessaires, elles exigent que ces nombres soient assez proche de 1 , mais la condition (6) est beaucoup plus faible que la condition (1). Dans les théorèmes de Yu Kunrui, on est obligé d'introduire un nombre premier $q$ vérifiant la condition (8); ce nombre premier $q$ dépend de $p, f$ et surtout des nombres $\alpha_{1}, \alpha_{2}$, et pour une minoration effective, il reste encore à montrer son existence et à le majorer en termes de $p, f$ et $\alpha_{1}, \alpha_{2}$ (Yu Kunrui a enlevé le paramètre $q$ du théorème 1 et du théorème 2 dans son article [10]). Cependant, le corollaire 1.1 donne une majoration d'une forme très simple qui fait intervenir beaucoup moins de paramètres. 


\section{Dong Pingping}

Notons d'ailleurs que le lemme de Liouville (sect. 2, lemme 2.4) donne la majoration suivante.

Corollaire 1.2. - On a

$$
\operatorname{ord}_{\mathfrak{p}} \Lambda \leq \frac{\mathrm{e} D}{\log p}\left\{\log 2+\left(a_{1}+a_{2}\right) B\right\} .
$$

Ce résultat n'exige aucune restriction sur les nombres algébriques $\alpha_{1}, \alpha_{2}$.

En imposant certaines relations sur $a_{1}, a_{2}$ et $B$, on pourra faire quelques comparaisons entre ces résultats : les deux théorèmes de Yu Kunrui (quand $p$ ne divise pas $\left.\left(b_{1}, b_{2}\right)\right)$, le corollaire 1.1 et le corollaire 1.2.

On distingue ici les quatre cas suivants :

$$
\begin{array}{llll}
\text { I } & a_{1} \ll 1, & a_{2} \ll 1 \\
\text { II } & B \ll a_{1}, & B \ll a_{2} \\
\text { III } & B \ll a_{1}, & B \ll a_{2}, \quad \log a_{1} \text { et } \log a_{2} \ll \log B \\
\text { IV } & B \ll 1 . &
\end{array}
$$

\begin{tabular}{|c|c|c|c|c|}
\hline $\operatorname{ord}_{\mathfrak{p}} \Lambda \ll$ & $\begin{array}{l}\text { Premier théorème } \\
\text { de Yu, } p \nmid\left(b_{1}, b_{2}\right)\end{array}$ & $\begin{array}{l}\text { Deuxième théorème } \\
\text { de Yu, } p \nmid\left(b_{1}, b_{2}\right)\end{array}$ & Corollaire 1.1 & Corollaire 1.2 \\
\hline I & $\begin{array}{l}D^{4} \log (4 D) \times \\
\quad \times(\log B+\log D)\end{array}$ & $D^{4}(\log B+\log D)^{2}$ & $D^{4} \log ^{2} B$ & $D B$ \\
\hline II & $\begin{array}{l}D^{4} a_{1} a_{2} \log (4 D) \\
(\log (4 D)+ \\
\left.+\log \max \left(1, a_{1} a_{2}\right)\right)\end{array}$ & $D^{4} a_{1} a_{2} \log ^{2}(4 D)$ & $D^{4} a_{1} a_{2} \log ^{2} B$ & $D\left(a_{1}+a_{2}\right) B$ \\
\hline III & $\begin{array}{c}D^{4} a_{1} a_{2} \log (4 D) \\
(\log B+\log D)\end{array}$ & $D^{4} a_{1} a_{2} \log ^{2}(4 D)$ & $D^{4} a_{1} a_{2} \log ^{2} B$ & $D\left(a_{1}+a_{2}\right) B$ \\
\hline $\operatorname{ord}_{\mathfrak{p}} \Lambda \ll$ & $\begin{array}{l}\text { Premier théorème } \\
\text { de Yu }\end{array}$ & $\begin{array}{l}\text { Deuxième théorème } \\
\text { de Yu }\end{array}$ & Corollaire 1.1 & Corollaire 1.2 \\
\hline IV & $\begin{array}{l}D^{4} a_{1} a_{2} \log (4 D) \\
(\log (4 D)+ \\
\left.+\log \max \left(1, a_{1}, a_{2}\right)\right)\end{array}$ & $D^{4} a_{1} a_{2} \log ^{2}(4 D)$ & $D^{4} a_{1} a_{2}$ & $D\left(a_{1}+a_{2}\right)$ \\
\hline
\end{tabular}

Dans ces cas, les quatres résultats possèdent quelquefois des formes plus simples qui sont exprimées dans le tableau ci-dessous.

Dans les majorations données par les théorèmes de Yu Kunrui, les «-constantes ne dépendent que de $p, \mathrm{e}, f, q$ et des $\ll$-constantes paraissant 
dans (9) cependant, dans les majorations données par les corollaires 1.1 et 1.2 , les «-constantes ne dépendent que de $p$, e et des «-constantes paraissant dans (9).

Il est difficile de dire quand mon résultat (corollaire 1.1) est meilleur en tenant compte en même temps des grandeurs et des constantes que ceux de Yu Kunrui, cependant dans les deux cas suivants, mon résultat est fréquemment meilleur que les siens :

1) en ce qui concerne la dépendance en $p$, quand $f \geq 3$;

2) en ce qui concerne la dépendance en $D$, quand $D$ est grand par rapport à $a_{1}, a_{2}$ et $B$.

\section{Lemmes auxiliaires}

Dans cette section nous donnons quelques lemmes auxiliaires qui nous seront utiles plus loin. Nous conservons dans toutes les sections suivantes les notations $\left(\mathbb{K}, \mathfrak{p}, p, f, \mathrm{e}, \nu,|\cdot|\right.$, ainsi que $\left.\alpha_{1}, \alpha_{2}, b_{1}, b_{2}, \Lambda\right)$ qui ont été introduites aux paragraphe 1 .

Quand $P$ est un élément de l'anneau $\mathbb{Z}\left[X_{1}, \ldots, X_{q}\right]$, on désigne par $L(P)$ sa longeur, somme des valeurs absolues usuelles de ces coefficients. Quand $G$ est une fonction analytique dans le disque $|z| \leq R$ de $\mathbb{C}_{p}$, avec $R \geq 0$, on désigne par $|G|_{R}$ la quantité $\sup _{|z| \leq R}|G|$.

Lemme 2.1 (lemme de Siegel). - Soient $\alpha_{1}, \ldots, \alpha_{q}$ des éléments de $\mathbb{K}$ et

$$
P_{i j} \in \mathbb{Z}\left[X_{1}, \ldots, X_{q}\right], \quad 1 \leq i \leq \nu, 1 \leq j \leq \mu,
$$

des polynômes (non tous nuls) de degré au plus $N_{j h}$ par rapport à $X_{h}$ (pour $1 \leq h \leq q)$. On définit

$$
L_{j}=\sum_{i=1}^{\nu} L\left(P_{i j}\right)
$$

et

$$
\gamma_{i j}=P_{i j}\left(\alpha_{1}, \ldots, \alpha_{q}\right), \quad 1 \leq i \leq \nu, 1 \leq j \leq \mu .
$$

Si $\nu>\mu D$, alors il existe des entiers rationnels $x_{1}, \ldots, x_{\nu}$ non tous nuls, tels que

$$
\sum_{i=1}^{\nu} \gamma_{i j} x_{i}=0, \quad 1 \leq j \leq \mu
$$




\section{Dong Pingping}

et

$$
\max \left\|x_{i}\right\| \leq\left(2^{\mu}\left(V_{1} \ldots V_{\mu}\right)^{D}\right)^{\frac{1}{\nu-\mu D}}
$$

$o \grave{u}$

$$
V_{j}=L_{j} e^{\sum_{h=1}^{q} N_{j h} h\left(\alpha_{h}\right)}
$$

Démonstration. - Voir [3].

Lеммe 2.2. - Soit $G$ une fonction analytique sur le disque $|z| \leq R$ de $\mathbb{C}_{p}$ qui possède $s$ zéros dans le disque $|z| \leq r(0<r<R)$. Alors

$$
|G|_{r} \leq|G|_{R}\left(\frac{r}{R}\right)^{s}
$$

Démonstration. - Voir [4].

LEMme 2.3. - Soit $F$ une fonction analytique sur le disque $|z| \leq R$ de $\mathbb{C}_{p}$ et soient $z_{1}, \ldots, z_{s} \in \mathbb{C}_{p}$ deux à deux distincts, avec $\left|z_{i}\right| \leq r, i=1, \ldots, s$ $(0<r<R)$. Alors

$$
|F|_{r} \leq \max \left\{|F|_{R}\left(\frac{r}{R}\right)^{s}, \frac{r^{s-1}}{\Delta}\left|F\left(z_{i}\right)\right|, i=1, \ldots, s\right\}
$$

où

$$
\Delta=\min _{1 \leq i \leq s} \prod_{j \neq i}\left|z_{i}-z_{j}\right|
$$

Démonstration. - La fonction

$$
G=F-\sum_{i=1}^{s} F\left(z_{i}\right) \prod_{j \neq i} \frac{z-z_{j}}{z_{i}-z_{j}}
$$

est analytique sur $|z| \leq R$ et possède $z$ zéros $z_{1}, \ldots, z_{s}$ dans le disque $|z| \leq r$; le lemme 2.2 dit alors

$$
\begin{aligned}
&|G|_{r} \leq|G|_{R}\left(\frac{r}{R}\right)^{s} \\
& \leq\left(\frac{r}{R}\right)^{s} \max \left\{|F|_{R},\left|F\left(z_{i}\right)\right| \prod_{j \neq i}\left|\frac{z-z_{j}}{z_{i}-z_{j}}\right|_{R}, i=1, \ldots, s\right\} \\
& \leq \max \left\{|F|_{R}\left(\frac{r}{R}\right)^{s}, \frac{r^{s-1}}{\Delta}\left|F\left(z_{i}\right)\right|, i=1, \ldots, s\right\}, \\
&-202-
\end{aligned}
$$


Minoration de combinaisons linéaires de deux logarithmes $p$-adiques

donc

$$
\begin{aligned}
|F|_{r} & \leq \max \left\{|G|_{r},\left|F\left(z_{i}\right)\right| \prod_{j \neq i}\left|\frac{z-z_{j}}{z_{i}-z_{j}}\right|_{r}, i=1, \ldots, s\right\} \\
& \leq \max \left\{|G|_{r}, \frac{r^{s-1}}{\Delta}\left|F\left(z_{i}\right)\right|, i=1, \ldots, s\right\},
\end{aligned}
$$

d'où la conclusion.

Lemme 2.4 (lemme de Liouville). - Soient $\alpha_{1}, \ldots, \alpha_{q} \in \mathbb{K}$ et soit $P \in \mathbb{Z}\left[X_{1}, \ldots, X_{q}\right]$ de degré au plus $N_{h}$ par rapport à $X_{h}(1 \leq h \leq q)$, tels que $\gamma=P\left(\alpha_{1}, \ldots, \alpha_{q}\right) \neq 0$. Alors :

a) pour toute valeur absolue archimédienne $v$ sur $\mathbb{K}$, on a

$$
|\gamma|_{v} \geq L(P)^{-(D-1)} e^{-D \sum_{h=1}^{q} N_{h} h\left(\alpha_{h}\right)}
$$

b) pour toute valeur absolue non archimédienne $v$ sur $\mathbb{K}$, on a

$$
|\gamma|_{v} \geq L(P)^{-D} e^{-D \sum_{h=1}^{q} N_{h} h\left(\alpha_{h}\right)}
$$

Démonstration. - Si $v$ est une valeur absolue archimédienne sur $\mathbb{K}$, on a

$$
|\gamma|_{v} \geq L(P) \prod_{h=1}^{q} \max \left(1,\left|\alpha_{h}\right|_{v}\right)^{N_{h}}
$$

et si $v$ est une valeur absolue non archimédienne sur $\mathbb{K}$, on a

$$
|\gamma|_{v} \geq \prod_{h=1}^{q} \max \left(1,\left|\alpha_{h}\right|_{v}\right)^{N_{h}}
$$

Comme $\gamma \neq 0$, on a la formule du produit

$$
\prod_{v}|\gamma|_{v}^{\sigma_{v}}=1
$$

où $v$ parcourt toutes les valeurs absolues sur $\mathbb{K}$ et $\sigma_{v}$ est le degré local de $v$.

Soit $v_{0}$ une valeur absolue sur $\mathbb{K}$ telle que $|\gamma|_{v_{0}} \leq 1$, alors

$$
|\gamma|_{v_{0}} \leq|\gamma|_{v_{0}}^{\sigma_{v_{0}}}=\frac{1}{\prod_{v \neq v_{0}}|\gamma|_{v}^{\sigma_{v}}}
$$




\section{Dong Pingping}

Si $v_{0}$ est archimédienne

$$
\begin{aligned}
|\gamma|_{v_{0}} & \geq L(P)^{-(D-1)} \prod_{h=1}^{q}\left(\prod_{v \neq v_{0}} \max \left(1,\left|\alpha_{h}\right|_{v}\right)^{\sigma_{v}}\right)^{-N_{h}} \\
& \geq L(P)^{-(D-1)} \prod_{h=1}^{q}\left(\prod_{v} \max \left(1,\left|\alpha_{h}\right|_{v}\right)^{\sigma_{v}}\right)^{-N_{h}} \\
& =L(P)^{-(D-1)} e^{-D \sum_{h=1}^{q} N_{h} h\left(\alpha_{h}\right)} .
\end{aligned}
$$

Si $v_{0}$ est non archimédienne

$$
\begin{aligned}
|\gamma|_{v_{0}} & \geq L(P)^{-(D)} \prod_{h=1}^{q}\left(\prod_{v \neq v_{0}} \max \left(1,\left|\alpha_{h}\right|_{v}\right)^{\sigma_{v}}\right)^{-N_{h}} \\
& \leq L(P)^{-D} \prod_{h=1}^{q}\left(\prod_{v} \max \left(1,\left|\alpha_{h}\right|_{v}\right)^{\sigma_{v}}\right)^{-N_{h}} \\
& =L(P)^{-(D)} e^{-D \sum_{h=1}^{q} N_{h} h\left(\alpha_{h}\right)} .
\end{aligned}
$$

Lemme 2.5. - Soient $\alpha, \beta, \gamma \in \mathbb{C}_{p}$ avec $\nu(\alpha-1)>1 /(p-1)$, $\nu(\beta-1)>1 /(p-1)$ et $\nu(\gamma) \geq 0$, on $a$

$$
|\log \alpha-\log \beta|=|\alpha-\beta|
$$

et

$$
\left|\alpha^{\gamma}-\beta^{\gamma}\right|=|\gamma||\alpha-\beta|
$$

Démonstration. - On sait que l'on a

$$
\log \alpha=\sum_{n=1}^{\infty}(-1)^{n-1} \frac{(\alpha-1)^{n}}{n} \text { et } \log \beta=\sum_{n=1}^{\infty}(-1)^{n-1} \frac{(\beta-1)^{n}}{n}
$$

donc

$$
\begin{aligned}
& \log \alpha-\log \beta= \sum_{n=1}^{\infty}(-1)^{n-1} \frac{(\alpha-1)^{n}-(\beta-1)^{n}}{n} \\
&=(\alpha-\beta) \sum_{n=1}^{\infty}(-1)^{n-1} \frac{1}{n} \sum_{i+j=n-1}^{\infty}(\alpha-1)^{i}(\beta-1)^{j} \\
&-204-
\end{aligned}
$$


Pour $n \geq 2$ et $i+j=n-1$, on a

$$
\begin{aligned}
\nu\left(\frac{1}{n}(\alpha-1)^{i}(\beta-1)^{j}\right) & =i \nu(\alpha-1)+j \nu(\beta-1)-\nu(n)> \\
& >\frac{n-1}{p-1}-\nu(n) \geq \\
& \geq \frac{n-1}{p-1}-\nu(n !) \geq 0
\end{aligned}
$$

alors

$$
\nu\left[\sum_{n=1}^{\infty}(-1)^{n-1} \frac{1}{n} \sum_{i+j=n-1}(\alpha-1)^{i}(\beta-1)^{j}\right]=0,
$$

d'où (10).

Comme $\log \alpha^{\gamma}=\gamma \log \alpha$ et $\log \beta^{\gamma}=\gamma \log \beta$, en utilisant (10), on trouve

$$
\begin{aligned}
\left|\alpha^{\gamma}-\beta^{\gamma}\right| & =\left|\log \left(\alpha^{\gamma}\right)-\log \left(\beta^{\gamma}\right)\right|=|\gamma||\log \alpha-\log \beta| \\
& =|\gamma||\alpha-\beta| .
\end{aligned}
$$

Soient $\alpha, \beta, \gamma$ des nombres complexes, $\alpha \gamma \neq 0$ et soient $L, M, U, V$ des entiers positifs. On veut démontrer que, sous des hypothèses convenables, si un polynôme $P \in \mathbb{C}[X, Y]$ non nul est de degré au plus $L$ et $M$ par rapport à $X$ et $Y$ respectivement, alors, au moins un des nombres

$$
P\left(u+v \beta, \alpha^{u} \gamma^{v}\right), \quad-U \leq u \leq U,-V \leq v \leq V,
$$

$(u, v) \in \mathbb{Z}^{2}$ est non nul.

Lemme 2.6. - Soient $U_{1}, U_{2}, V_{1}, V_{2}$ des entiers positifs. Posons $U=$ $U_{1}+U_{2}$ et $V=V_{1}+V_{2}$.

On suppose :

i) les points $u+v \beta,\left(-U_{1} \leq u \leq U_{1},-V_{1} \leq v \leq V_{1}\right)$ sont deux à deux distincts, et $\left(2 U_{1}+1\right)\left(2 V_{1}+1\right)>L$;

ii) $\operatorname{card}\left\{\alpha^{u} \gamma^{v} ;-U_{1} \leq u \leq U_{1},-V_{1} \leq v \leq V_{1}\right\}>M$;

iii) $\operatorname{card}\left\{u+v \beta ;-U_{2} \leq u \leq U_{2},-V_{2} \leq v \leq V_{2}\right\}>2 L M$. 


\section{Dong Pingping}

Alors, au moins un des nombres

$$
P\left(u+v \beta, \alpha^{u} \gamma^{v}\right),-U \leq u \leq U,-V \leq v \leq V
$$

est non nul.

Démonstration. - Voir [6].

Pour la construction de la fonction auxiliaire on introduit des polynômes $\Delta_{h},(h \in \mathbb{Z}, h \geq 0)$, définis par $\Delta_{0}=1$ et

$$
\Delta_{h}(z)=\left\{\begin{array}{l}
\frac{1}{h !}\left(z-\frac{h-1}{2}\right) \cdots(z-1) z(z+1) \cdots\left(z+\frac{h-1}{2}\right) \\
\frac{1}{h !}\left(z-\frac{h-2}{2}\right) \cdots(z-1) z(z+1) \cdots\left(z+\frac{h-2}{2}\right)\left(z+\frac{h}{2}\right) \\
\text { si } h \text { est pair. }
\end{array}\right.
$$

Pour $z$ dans $\mathbb{Z}$ on a $\Delta_{h}(z) \in \mathbb{Z}$.

Lemme 2.7. - Soient $h \geq 0$ et $z \in \mathbb{R}$. On a

$$
\left\|\Delta_{h}(z)\right\| \leq 2 \frac{E^{h}}{h !} \quad \text { avec } \quad E=\max \left(\|z\|, \frac{h}{2}\right) .
$$

Démonstration. - Considérons d'abord le cas où $h$ est impair. Pour $1 \leq i \leq(h-1) / 2$, on a $\left\|z^{2}-i^{2}\right\| \leq E^{2}$, donc

$$
\left\|\Delta_{h}(z)\right\|=\frac{1}{h !}\|z\| \prod_{i=1}^{(h-1) / 2}\left\|z^{2}-i^{2}\right\| \leq \frac{E \cdot E^{h-1}}{h !}=\frac{E^{h}}{h !} .
$$

Supposons maintenant que $h$ est pair; pour $1 \leq i \leq(h-2) / 2$, on a $\left\|z^{2}-i^{2}\right\| \leq E^{2}$, donc

$$
\left\|\Delta_{h}(z)\right\|=\frac{1}{h !}\|z\|\left\|z+\frac{h}{2}\right\| \prod_{i=1}^{(h-1) / 2}\left\|z^{2}-i^{2}\right\| \leq \frac{2 E^{2} \cdot E^{h-2}}{h !}=2 \frac{E^{h}}{h !}
$$




\section{Estimation d'un produit}

Lemme 3.1. - Pour un nombre premier $p$ fixé, on désigne par $|\cdot|$ la valeur absolue $p$-adique sur $\mathbb{Q}$

$$
|x|=p^{-\nu_{p}(x)}, \quad x \in \mathbb{Q} .
$$

Soient $b_{1}, b_{2} \in \mathbb{Z}$ et $\neq 0, p \nmid b_{2},\left\|b_{1}\right\|,\left\|b_{2}\right\| \leq B, B \geq 2$ et soit $\beta=b_{1} / b_{2}$. Soient $U, V$ deux entiers $\geq 2$, on suppose que les points

$$
u+v \beta, \quad(u, v) \in \mathbb{Z}^{2}, \quad-U \leq u \leq U, \quad-V \leq v \leq V
$$

sont deux à deux distincts, et on note

$$
\begin{gathered}
\Gamma=\left\{u+v \beta,(u, v) \in \mathbb{Z}^{2},-U \leq u \leq U,-V \leq v \leq V\right\} \\
\Delta=\min _{\gamma \in \Gamma} \prod_{\substack{\gamma^{\prime} \in \Gamma \\
\gamma \neq \gamma}}\left|\gamma^{\prime}-\gamma\right| .
\end{gathered}
$$

On $a$

$$
\begin{aligned}
-\log \Delta= & 4\left(\frac{1}{p-1}+\frac{1}{p^{2}}\right) U V \log p+2 U \log 2 B(U+V)+ \\
& +2 V\left(\log B+\frac{\log p}{p-1}\right)
\end{aligned}
$$

Démonstration. - Soit $\gamma_{0}=u_{0}+v_{0} \beta \in \Gamma\left(-U \leq u_{0} \leq U,-V \leq v_{0}\right.$ $\leq V)$ un point où le minimum de $\prod\left|\gamma-\gamma_{0}\right|$ est atteint, on a pour $\gamma \in \Gamma$ et $\neq \gamma_{0}$

$$
\begin{gathered}
\Delta=\prod_{\substack{\gamma \in \Gamma \\
\gamma \neq \gamma_{0}}}\left|\gamma-\gamma_{0}\right|=\prod_{\begin{array}{c}
-U \leq u \leq U \\
-V \leq v \leq V \\
(u, v) \neq\left(u_{0}, v_{0}\right)
\end{array}}\left|\left(u-u_{0}\right)+\left(v-v_{0}\right) \beta\right| \\
=\prod_{\substack{-U \leq u \leq U \\
-V \leq v \leq V \\
(u, v) \neq\left(u_{0}, v_{0}\right)}}\left|b_{2}\left(u-u_{0}\right)+b_{1}\left(v-v_{0}\right)\right|=\prod_{\substack{-U-u_{0} \leq s \leq U-u_{0} \\
-V-v_{0} \leq t \leq V-v_{0} \\
(s, t) \neq 0}}\left|b_{2} s+b_{1} t\right| .
\end{gathered}
$$




\section{Dong Pingping}

Soit $f \geq 0$ l'entier défini par $p^{f} \| b_{1}, b_{1}=p^{f} b, p \nmid b$, on voit que

$$
\Delta=\left|A_{1}\right|\left|A_{2}\right|\left|A_{3}\right|
$$

où

$$
\begin{aligned}
& A_{1}=\prod_{\substack{-U-u_{0} \leq s \leq U-u_{0} \\
s \equiv 0\left(p^{f}\right) \\
s \neq 0}} \prod_{-V-v_{0} \leq t \leq V-v_{0}}\left(b_{2} s+b_{1} t\right),
\end{aligned}
$$

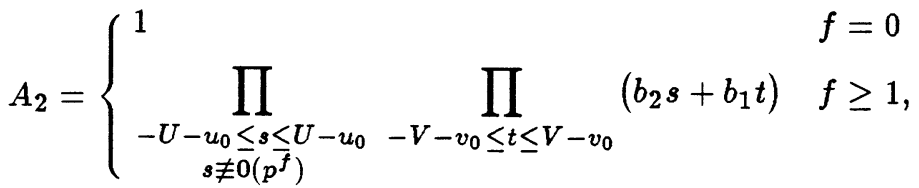

$$
\begin{aligned}
& A_{3}=b_{1}^{2 V} \prod_{\substack{-V-v_{0} \leq t \leq V-v_{0} \\
t \neq 0}} t .
\end{aligned}
$$

Il n'existe que $\left[2 U / p^{f}\right]$ entiers $s$ tel que

$$
-U-u_{0} \leq s \leq U-u_{0}, \quad s \equiv 0\left(p^{f}\right), \quad s \neq 0 .
$$

Pour un tel entier $s$, on a

$$
\prod_{-V-v_{0} \leq t \leq V-v_{0}}\left(b_{2} s+b_{1} t\right)=p^{(2 V+1) f} \prod_{-V-v_{0} \leq t \leq V-v_{0}}\left(b_{2} s p^{-f}+b t\right) .
$$

Pour $-V-v_{0} \leq t \leq V-v_{0}$, on a

$$
\log _{p}\left\|b_{2} p^{-f}+b t\right\| \leq(\log 2 B(U+V) / \log p)-f
$$

et pour $l \geq 1$, dans l'intervalle $\left[-V-v_{0}, V-v_{0}\right]$, il n'existe que $\left[\left(2 V / p^{l}\right)+1\right]$ entiers $t$ tels que $b_{2} s p^{-f}+b t \equiv 0\left(\bmod p^{l}\right)$, donc

$$
\begin{aligned}
\nu_{p}\left[\prod_{-V-v_{0} \leq t \leq V-v_{0}}\left(b_{2} s p^{-f}+b t\right)\right] & \leq \sum_{1 \leq l \leq(\log 2 B(U+V) / \log p)-f}\left(\frac{2 V}{p^{l}}+1\right) \\
& \leq \frac{2 V}{p-1}+\frac{\log 2 B(U+V)}{\log p}-f, \\
& -208-
\end{aligned}
$$


Minoration de combinaisons linéaires de deux logarithmes $p$-adiques

$$
\begin{aligned}
& \nu_{p}\left[\prod_{-V-v_{0} \leq t \leq V-v_{0}}\left(b_{2} s+b_{1} t\right)\right]=(2 V+1) f+\nu_{p}\left[\prod_{-V-v_{0} \leq t \leq V-v_{0}}\left(b_{2} s p^{-f}+b t\right)\right] \\
& \leq 2 V\left(f+\frac{1}{p-1}\right)+\frac{\log 2 B(U+V)}{\log p}, \\
& -\log \left|A_{1}\right|=\nu_{p}\left(A_{1}\right) \log p \leq \\
& \leq \frac{2 U}{p^{f}}\left[2 V\left(f+\frac{1}{p-1}\right)+\frac{\log 2 B(U+V)}{\log p}\right] \log p \\
& =\frac{2 U}{p^{f}}\left[2 V\left(f+\frac{1}{p-1}\right) \log p+\log 2 B(U+V)\right] \text {. }
\end{aligned}
$$

Dans le cas $f \geq 1$

$$
\begin{aligned}
& \nu_{p}\left[\prod_{\substack{U-u_{0} \leq s \leq U-u_{0} \\
s \neq 0\left(p^{f}\right)}} s\right] \leq \sum_{l=1}^{f-1}\left[\frac{2 U}{p^{l}}\right] \\
& \leq 2 U \frac{1-p^{-(f-1)}}{p-1} \\
& \nu_{p}\left(A_{2}\right)=(2 V+1) \nu_{p}\left[\prod_{\substack{-u_{0} \leq s \leq U-u_{0} \\
s \neq 0\left(p^{f}\right)}} s\right] \\
& \leq 2 U(2 V+1) \frac{1-p^{-(f-1)}}{p-1} \\
& -\log \left|A_{2}\right|=\nu_{p}\left(A_{2}\right) \log p \leq 2 U(2 V+1) \frac{1-p^{-(f-1)}}{p-1} \log p \\
& \nu_{p}\left[\prod_{\substack{v_{0} \leq t \leq V-v_{0} \\
t \neq 0}}\right] \leq \frac{2 V}{p-1} \\
& \nu_{p}\left(A_{3}\right) \leq 2 V\left(f+\frac{1}{p-1}\right), \\
& -\log \left|A_{3}\right|=\nu_{p}\left(A_{3}\right) \log p=2 V\left(f+\frac{1}{p-1}\right) \log p .
\end{aligned}
$$




\section{Dong Pingping}

Si $f=0$, don déduit de (12), (13), (15)

$$
-\log \Delta \leq \frac{4 \log p}{p-1} U V+2 U \log 2 B(U+V)+2 V \frac{\log p}{p-1} .
$$

Supposons $f \geq 1$, on déduit de (12), (13), (14), (15)

$$
\begin{aligned}
&-\log \Delta \leq 4 U V\left[\frac{1}{p^{f}}\left(f+\frac{1}{p-1}\right)+\frac{1-p^{-(f-1)}}{p-1}\right] \log p+ \\
&+2 U\left[\frac{1}{p^{f}} \log 2 B(U+V)+\frac{\log p}{p-1}\right]+2 V\left(f+\frac{1}{p-1}\right) \log p, \\
& \frac{1}{p^{f}}\left(f+\frac{1}{p-1}\right)+\frac{1-p^{-(f-1)}}{p-1}=\frac{1}{p-1}+\frac{f-1}{p^{f}} \leq \frac{1}{p-1}+\frac{1}{p^{2}}, \\
& \frac{1}{p^{f}} \log 2 B(U+V)+\frac{\log p}{p-1} \leq \log 2 B(U+V), \\
&\left(f+\frac{1}{p-1}\right) \log p \leq \log B+\frac{\log p}{p-1}
\end{aligned}
$$

d'où le résultat désiré. $\square$

Lemme 3.2. - Sous les conditions du lemme 3.1, on a

$$
-\log \Delta \leq \frac{4 \log p}{p-1} U V+2 U \log 2 B(U+V)+2 V \frac{\log p}{p-1} .
$$

Démonstration. - Nous conservons les notations et les conclusions de la démonstration du lemme 3.1. On a

$$
\Delta=\left|A_{1}^{\prime}\right|\left|A_{2}^{\prime}\right|
$$

où

$$
\begin{aligned}
& A_{1}^{\prime}=\prod_{\substack{-v_{0} \leq t \leq V-v_{0} \\
t \neq 0}} \prod_{-U-u_{0} \leq s \leq U-u_{0}}\left(b_{2} s+b_{1} t\right) \\
& A_{2}^{\prime}=\prod_{\substack{-U-u_{0} \leq s \leq U-u_{0} \\
s \neq 0}} s .
\end{aligned}
$$


Minoration de combinaisons linéaires de deux logarithmes p-adiques

$\mathrm{Si}-V-v_{0} \leq t \leq V-v_{0}, t \neq 0,-U-u_{0} \leq s \leq U-u_{0}$

$$
\log _{p}\left\|b_{2} s+b_{1} t\right\| \leq \frac{\log 2 B(U+V)}{\log p} .
$$

$\mathrm{Si}-V-v_{0} \leq t \leq V-v_{0}, t \neq 0$, pour $l \geq 1$, dans l'intervalle [ $-U-u_{0}, U-$ $\left.u_{0}\right]$, il n'existe que $\left[\left(2 U / p^{l}\right)+1\right]$ entiers $s$ tels que

$$
b_{2} s+b_{1} t \equiv 0 \quad\left(p^{l}\right)
$$

donc

$$
\begin{gathered}
\nu_{p}\left[\prod_{-U-u_{0} \leq s \leq U-u_{0}}\left(b_{2} s+b_{1} t\right)\right] \leq \sum_{1 \leq l \leq(\log 2 B(U+V) / \log p)}\left(\frac{2 U}{p^{l}}+1\right) \\
\leq \frac{2 U}{p-1}+\frac{\log 2 B(U+V)}{\log p} \\
\nu_{p}\left(A_{1}^{\prime}\right)=\sum_{-V-v_{0} \leq t \leq V-v_{0}} \nu_{t \neq 0}\left[\prod_{-U-u_{0} \leq s \leq U-u_{0}}\left(b_{2} s+b_{1} t\right)\right] \\
\leq 2 V\left(\frac{2 U}{p-1}+\frac{\log 2 B(U+V)}{\log p}\right) \\
-\log \left|A_{1}^{\prime}\right|=\nu_{p}\left(A_{1}^{\prime}\right) \log p \leq \frac{4 \log p}{p-1} U V+2 V \log 2 B(U+V) \\
\nu_{p}\left(A_{2}^{\prime}\right) \leq \sum_{l \geq 1}\left[\frac{2 U}{p^{l}}\right] \leq \frac{2 U}{p-1} \\
-\log \left|A_{2}^{\prime}\right|=\nu_{p}\left(A_{2}^{\prime}\right) \log p \leq \frac{2 \log p}{p-1} U
\end{gathered}
$$

La conclusion est obtenue en utilisant (16), (17) et (18).

Corollaire 3.3. - Sous les conditions du lemme 3.1. On a $-\log \Delta \leq 4\left(\frac{1}{p-1}+\frac{1}{p^{2}}\right) U V \log p+$

$$
+2 \max (U, V)\left[2 \log B+\log \min (U, V)+2 \log 2+\frac{\log p}{p-1}\right] .
$$




\section{Dong Pingping}

\section{Démonstration}

Cas 1. $U \leq V$ On utilise le lemme 3.1 et l'inégalité $U \log V \leq V \log U$. On a

$$
\begin{aligned}
-\log \Delta \leq & 4\left(\frac{1}{p-1}+\frac{1}{p^{2}}\right) U V \log p+2 U \log 4 B V+2 V\left(\log B+\frac{\log p}{p-1}\right) \\
=4 & \left(\frac{1}{p-1}+\frac{1}{p^{2}}\right) U V \log p+2 U(\log B+\log V+2 \log 2)+ \\
& +2 V\left(\log B+\frac{\log p}{p-1}\right) \\
\leq & 4\left(\frac{1}{p-1}+\frac{1}{p^{2}}\right) U V \log p+ \\
& +2 V\left(2 \log B+\log U+2 \log 2+\frac{\log p}{p-1}\right) .
\end{aligned}
$$

Cas $2 V \leq U$ On utilise le lemme 3.2 et l'inégalité $V \log U \leq U \log V$. On a

$$
\begin{aligned}
-\log \Delta & \leq \frac{4 \log p}{p-1} U V+2 V \log 4 B U+2 U \frac{\log p}{p-1} \\
& =\frac{4 \log p}{p-1} U V+2 V(\log B+\log U+2 \log 2)+2 U \frac{\log p}{p-1} \\
& \leq \frac{4 \log p}{p-1} U V+2 U\left(\log B+\log V+2 \log 2+\frac{\log p}{p-1}\right)
\end{aligned}
$$

\section{Le résultat principal}

Dans cette section nous donnons le résultat principal : le théorème 4.1. Tout d'abord, nous plongeons le corps valué $(\mathbb{K}, \nu)$ dans $\mathbb{C}_{p}$ comme un de ses sous-corps valués.

THÉORÈME 4.1. - On utilise les paramètres $a_{1}, a_{2}, B$ qui ont été définis dans (2) et (3), et on fait l'hypothèse (1).

Pour $p \geq 3$, on a

$$
\begin{gathered}
\log \frac{|\Lambda|}{p} \geq-T \\
-212-
\end{gathered}
$$


avec

$$
\begin{aligned}
T= & \frac{2500}{\log ^{3} p}\left[\left(\frac{p}{p-1}+\frac{1}{p^{2}}\right) p^{2}+0.1715 p\right] D^{4} a_{1} a_{2} \log ^{2} B+ \\
& -\frac{1111.22}{\log ^{2} p}\left(1+0.0587 \frac{1}{p}\right) D^{4} a_{1} a_{2} \log B+ \\
& +\left\{\frac{84.9175 p^{2}}{\log ^{2} p}\left[1+(0.0488+0.0001 \log \zeta) \frac{1}{p}\right]+\frac{2.4235 \cdot p}{\log p}\left(1+\frac{0.0697}{p}\right)\right. \\
& \left.+\frac{63.315 \cdot p}{\log ^{2} p}\left(1+0.7214 \frac{\log p}{p-1}\right)\left(1+\frac{0.0402}{p}\right)\right\} D^{3}\left(a_{1}+a_{2}\right) \log B
\end{aligned}
$$

$o u ̀$

$$
\zeta=\max \left(1, \frac{e}{2 \log p}\right)= \begin{cases}1.2371 \ldots & p=3 \\ 1 . & p \geq 5\end{cases}
$$

Pour $p=2$, on $a$

$$
\log \frac{|\Lambda|}{4} \geq-T
$$

$o \grave{u}$

$$
\begin{aligned}
T= & 37390.252 D^{4} a_{1} a_{2} \log ^{2} B-9724.9496 D^{4} a_{1} a_{2} \log B \\
& +1188.3801 D^{3}\left(a_{1}+a_{2}\right) \log B .
\end{aligned}
$$

Nous démontrerons la proposition suivante où une condition supplémentaire est imposée, puis nous déduirons le théorème 4.1 de cette proposition.

Proposition 4.2. - On suppose $\left(b_{1}, b_{2}\right)=1$.

Pour $p \geq 3$, on $a$

$$
\log \frac{|\Lambda|}{p} \geq-T
$$

Pour $p=2$, on $a$

$$
\log \frac{|\Lambda|}{4} \geq-T
$$

où $T$ est donné dans l'énoncé du théorème 4.1.

LEMME 4.3. - Si $1 \leq \log B \leq 2 \log p$, on $a$

$$
\log |\Lambda| \geq-0.5479 \frac{p^{2}}{\log ^{3} p} D a_{1} a_{2} \log ^{2} B .
$$




\section{Dong Pingping}

Démonstration. - Nous utilisons le lemme 2.4 (b)

$$
\begin{aligned}
\log |\Lambda| & \geq-D\left(\log 2+\left\|b_{1}\right\| a_{1}+\left\|b_{2}\right\| a_{2}\right) \geq-D\left[\log 2+B\left(a_{1}+a_{2}\right)\right] \\
& \geq-D\left[\log 2+\frac{p^{2}}{2 \log p}\left(a_{1}+a_{2}\right) \log B\right] \\
& \geq-D\left[\log 2+\frac{p^{2}}{2 \log ^{2} p} a_{1} a_{2} \log B\right] \\
& \geq-0.5434 \frac{p^{2}}{\log ^{2} p} D a_{1} a_{2} \log B
\end{aligned}
$$

Si $\log B \geq \log p$, on a

$$
\log |\Lambda| \geq-0.5434 \frac{p^{2}}{\log ^{3} p} D a_{1} a_{2} \log ^{2} B
$$

et si $\log B<\log p$, on a

$$
\begin{aligned}
\log |\Lambda| & \geq-D\left[\log 2+p\left(a_{1}+a_{2}\right)\right] \\
& \geq-\left[\frac{\log 2}{4 p^{2}}+\frac{\log p}{p}\right] \frac{p^{2}}{\log ^{2} p} D a_{1} a_{2} \log ^{2} B \\
& \geq-0.5479 \frac{p^{2}}{\log ^{3} p} D a_{1} a_{2} \log ^{2} B .
\end{aligned}
$$

Démonstration de la proposition 4.2.- Grâce au lemme 4.3 on peut supposer $\log B>2 \log p$.

Soient $c \geq 2, c_{0} \geq 1, c_{1} \geq 1 / \log p$ des nombres réels qui ne dépendent que de $p$ et qui satisfont

$$
\begin{aligned}
& c_{0}\left(2 c_{1}-\frac{1}{2 \log p}\right)>\left(2 c+\frac{1}{4 \log ^{2} p}\right)^{2} \\
& 2 \log ^{2} p \cdot c_{0} \geq c_{1} .
\end{aligned}
$$

On pose

$$
\eta=\frac{\left(2 c+\frac{1}{4 \log ^{2} p}\right)^{2}}{c_{0}\left(2 c_{1}-\frac{1}{2 \log p}\right)-\left(2 c+\frac{1}{4 \log ^{2} p}\right)^{2}}
$$


Minoration de combinaisons linéaires de deux logarithmes $p$-adiques

Soient $\chi_{1}, \chi_{2}, \chi$ des nombres positifs ne dépendant que de $p$, et satisfaisant

$$
\begin{aligned}
\chi_{1} & >\frac{1}{2 c}\left(\sqrt{\frac{c_{0}}{2 \log p}}+\frac{1}{4 \log ^{2} p}\right) \\
\chi_{2} & >\frac{1}{c}\left(\sqrt{c_{0} c_{1}}+\frac{1}{8 \log ^{2} p}\right) \\
\chi_{2} & \geq 1 \text { et } \chi_{1} \\
\chi & \geq \chi_{1}+\chi_{2}+\frac{1}{4 c \log ^{2} p} .
\end{aligned}
$$

On note $\mathbb{K}_{0}=\mathbb{Q}\left(\alpha_{1}, \alpha_{2}\right) \subset \mathbb{K}$, et $D_{0}=\left[\mathbb{K}_{0}: \mathbb{Q}\right] \leq D$. Nous écrivons

$$
\begin{aligned}
L_{0} & =\left[c_{0} D^{3} a_{1} a_{2} \log B\right] & L_{1} & =\left[c_{1} D \log B\right] \\
M_{1} & =\left[c D^{2} a_{2} \log B\right] & M_{2} & =\left[c D^{2} a_{1} \log B\right] \\
U_{1} & =\left[\chi_{1} c D^{2} a_{2} \log B\right] & V_{1} & =\left[\chi_{1} c D^{2} a_{1} \log B\right] \\
U_{2} & =\left[\chi_{2} c D^{2} a_{2} \log B\right] & V_{2} & =\left[\chi_{2} c D^{2} a_{1} \log B\right] \\
M_{1}^{*} & =\left[\chi c D^{2} a_{2} \log B\right] & M_{2}^{*} & =\left[\chi c D^{2} a_{1} \log B\right] \\
\mu= & & &
\end{aligned}
$$

Les inégalités suivantes nous seront fréquemment utiles

$$
\begin{aligned}
& \left\{\begin{array}{l}
L_{0}+1 \geq c_{0} D^{3} a_{1} a_{2} \log B \\
\left(2 c_{1}-\frac{1}{2 \log p}\right) D \log B \leq 2 L_{1}+1 \leq\left(2 c_{1}+\frac{1}{2 \log p}\right) D \log B
\end{array}\right. \\
& \left\{\begin{array}{c}
\left(2 c-\frac{1}{4 \log ^{2} p}\right) D^{2} a_{2} \log B \leq 2 M_{1}+1 \\
\leq\left(2 c+\frac{1}{4 \log ^{2} p}\right) D^{2} a_{2} \log B \\
\left(2 c-\frac{1}{4 \log ^{2} p}\right) D^{2} a_{1} \log B \leq 2 M_{2}+1 \\
\leq\left(2 c+\frac{1}{4 \log ^{2} p}\right) D^{2} a_{1} \log B
\end{array}\right.
\end{aligned}
$$




$$
\left\{\begin{array}{l}
2 U_{1}+1 \geq\left(2 \chi_{1} c-\frac{1}{4 \log ^{2} p}\right) D^{2} a_{2} \log B \\
2 V_{1}+1 \geq\left(2 \chi_{1} c-\frac{1}{4 \log ^{2} p}\right) D^{2} a_{1} \log B \\
2 U_{2}+1 \geq\left(2 \chi_{2} c-\frac{1}{4 \log ^{2} p}\right) D^{2} a_{2} \log B \\
2 V_{2}+1 \geq\left(2 \chi_{2} c-\frac{1}{4 \log ^{2} p}\right) D^{2} a_{1} \log B
\end{array}\right.
$$

Cas 1. Pour deux couples différents $(u, v),\left(u^{\prime}, v^{\prime}\right) \in \mathbb{Z}^{2}$ vérifiant $\|u\|$, $\left\|u^{\prime}\right\| \leq U_{1},\|v\|,\left\|v^{\prime}\right\| \leq V_{1}$, on a $\alpha_{1}^{p u} \alpha_{2}^{p v}=\alpha_{1}^{p u^{\prime}} \alpha_{2}^{p v^{\prime}}$ (si $p \geq 3$ ), $\alpha_{1}^{4 u} \alpha_{2}^{4 v}=\alpha_{1}^{4 u^{\prime}} \alpha_{2}^{4 v^{\prime}}($ si $p=2)$.

Soient $l_{1}=u-u^{\prime}, l_{2}=v-v^{\prime}$, on a $\alpha_{2}^{p l_{2}}(p>3), \alpha_{2}^{4 l_{1}}=\alpha_{2}^{4 l_{2}}(p=2)$.

Sans perte de généralité, on peut supposer que $l_{2} \neq 0$, on note $N=$ $b_{1} l_{2}-b_{2} l_{1}$. Alors :

$$
\begin{gathered}
\left(a_{1}+a_{2}\right) \log B \leq \frac{2}{p} a_{1} a_{2} B^{1 / 2} \\
\|N\| \leq B\left(\left\|l_{1}\right\|+\left\|l_{2}\right\|\right) \leq 2 B\left(U_{1}+V_{1}\right) \\
\leq 2 B \chi_{1} c D^{2}\left(a_{1}+a_{2}\right) \log B \leq \frac{4}{p} \chi_{1} c D^{2} a_{1} a_{2} B^{3 / 2} .
\end{gathered}
$$

Si $N \neq 0$

$$
\begin{aligned}
-\log |N| & \leq \log \|N\| \leq \log \frac{4 \chi_{1} c}{p}+\log \left(D^{2} a_{1} a_{2}\right)+\frac{3}{2} \log B \\
& =\log \frac{4 \chi_{1} c}{p}+2 \log D+\log \left(a_{1} a_{2}\right)+\frac{3}{2} \log B \\
& \leq\left\{\begin{array}{r}
\frac{1}{8 \log ^{3} p}\left[\log \frac{16 \chi_{1} c \log ^{2} p}{p}+\frac{2}{e}+3 \log p\right] D a_{1} a_{2} \log B, \\
\frac{1}{8 \log ^{3} 2}\left[\log \left(\chi_{1} c\right)+\frac{2}{e}+1+4 \log 2\right] D a_{1} a_{2} \log B, \quad \\
p=2
\end{array}\right.
\end{aligned}
$$

et si $\alpha_{1} \neq 1$ le lemme 2.4 (b) dit

$$
\log \left|\alpha_{1}-1\right| \geq-2 D a_{1} \geq \frac{1}{2 \log ^{2} p} D a_{1} a_{2} \log B .
$$


Minoration de combinaisons linéaires de deux logarithmes $p$-adiques

Maintenant, on utilise le lemme 2.5 , dans le cas $p \geq 3$, on a

$$
\begin{aligned}
|\Lambda| \geq\left|l_{2}\right||\Lambda| & =p\left|p l_{2}\right||\Lambda|=p\left|\alpha_{1}^{p b_{1} l_{2}}-\alpha_{2}^{p b_{2} l_{2}}\right| \\
& =p\left|\alpha_{1}^{p b_{1} l_{2}}-\alpha_{1}^{p b_{2} l_{1}}\right|=p\left|\alpha_{1}^{p N}-1\right| \\
& =|N|\left|\alpha_{1}-1\right| \quad(p \geq 3)
\end{aligned}
$$

et dans le cas $p=2$, on a

$$
\begin{aligned}
|\Lambda| \geq\left|l_{2}\right||\Lambda| & =4\left|4 l_{2}\right||\Lambda|=4\left|\alpha_{1}^{4 b_{1} l_{2}}-\alpha_{2}^{4 b_{2} l_{2}}\right| \\
& =4\left|\alpha_{1}^{4 b_{1} l_{2}}-\alpha_{1}^{4 b_{2} l_{1}}\right|=4\left|\alpha_{1}^{4 N}-1\right| \\
& =|N|\left|\alpha_{1}-1\right| \quad(p=2) .
\end{aligned}
$$

On voit d'abord que, dans tous les cas où $N \neq 0$ et $\alpha_{1} \neq 1$,

$$
\begin{aligned}
\log |\Lambda| & \geq \log |N|+\log \left|\alpha_{1}-1\right| \\
& \geq\left\{\begin{array}{r}
-\frac{1}{8 \log ^{3} p}\left[\log \frac{16 \chi_{1} c \log ^{2} p}{p}+\frac{2}{e}+7 \log p\right] \quad D a_{1} a_{2} \log B \\
-\frac{1}{8 \log ^{3} 2}\left[\log \left(\chi_{1} c\right)+\frac{2}{e}+1+8 \log 2\right] D a_{1} a_{2} \log B \\
p=3
\end{array}\right.
\end{aligned}
$$

Puisque $\left(b_{1}, b_{2}\right)=1$, on peut supposer que $p \nmid b_{2}$ et on pose $\beta=b_{1} / b_{2}$. Cas 2. Pour deux couples différents $(u, v),\left(u^{\prime}, v^{\prime}\right) \in \mathbb{Z}^{2}$, vérifiant $\|u\|$, $\left\|u^{\prime}\right\| \leq U_{2},\|v\|,\left\|v^{\prime}\right\| \leq V_{2}$, on a

$$
u+v \beta=u^{\prime}+v^{\prime} \beta .
$$

Alors $\beta=-\frac{u-u^{\prime}}{v-v^{\prime}}$ et, puisque $\left(b_{1}, b_{2}\right)=1$, on a

$$
\left\|b_{1}\right\| \leq\left\|u-u^{\prime}\right\| \leq 2 U_{2}, \quad\left\|b_{2}\right\| \leq\left\|v-v^{\prime}\right\| \leq 2 V_{2},
$$

on en déduit

$$
\left\|b_{1}\right\| a_{1}+\left\|b_{2}\right\| a_{2} \leq 2\left(U_{2} a_{1}+V_{2} a_{2}\right) \leq 4 \chi_{2} c D^{2} a_{1} a_{2} \log B .
$$

Comme $\Lambda \neq 0$, le lemme 2.4 (b) donne

$$
\begin{aligned}
\log |\Lambda| & \geq-D\left(\log 2+\left\|b_{1}\right\| a_{1}+\left\|b_{2}\right\| a_{2}\right) \\
& \geq-D\left(\log 2+4 \chi_{2} c D^{2} a_{1} a_{2} \log B\right) \\
& \geq-\left(\frac{\log 2}{8 \log ^{3} p}+4 \chi_{2} c\right) D^{3} a_{1} a_{2} \log B .
\end{aligned}
$$




\section{Dong Pingping}

Cas 3. $B c\left(a_{1}+a_{2}\right)<\left(c_{0} / 2\right) D a_{1} a_{2}$.

Comme $\Lambda \neq 0$, le lemme 2.4 (b) donne

$$
\begin{aligned}
\log |\Lambda| & \geq-D\left(\log 2+\left\|b_{1}\right\| a_{1}+\left\|b_{2}\right\| a_{2}\right) \\
& \geq-D\left(\log 2+B\left(a_{1}+a_{2}\right)\right) \\
& >-D\left(\log 2+\frac{c_{0}}{2 c} D a_{1} a_{2}\right) \\
& \geq-\left(\frac{\log 2}{4 \log ^{2} p}+\frac{c_{0}}{2 c}\right) D^{2} a_{1} a_{2} .
\end{aligned}
$$

Enfin on introduit l'hypothèse suivante :

Hypothèse (H). - Les points $u+v \beta,-M_{1} \leq u \leq M_{1},-M_{2} \leq v \leq M_{2}$ sont deux à deux distincts.

Proposition 4.4. - Sous l'hypothèse (H), on note

$$
\Gamma=\left\{u+b \beta \mid-M_{1} \leq u \leq M_{1},-M_{2} \leq v \leq M_{2}\right\}
$$

et

$$
\Delta=\min _{\gamma \in \Gamma} \prod_{\gamma^{\prime} \in \Gamma, \neq \gamma}\left|\gamma-\gamma^{\prime}\right|>0
$$

On a

$$
\begin{aligned}
-\log \Delta \leq & \left\{4\left(\frac{1}{p-1}+\frac{1}{p^{2}}\right) c^{2} \log p+\right. \\
& \left.+2\left[\frac{1}{\log p}+\frac{\log \left(4 c \log ^{2} p\right)}{4 \log ^{2} p}\right] c\right\} D^{4} a_{1} a_{2} \log ^{2} B+ \\
+ & 2\left(\log 4+\frac{\log p}{p-1}\right) c D^{2}\left(a_{1}+a_{2}\right) \log B
\end{aligned}
$$

Démonstration. - On utilise le corollaire 3.3 avec $U=M_{1}, V=M_{2}$, on voit

$$
\begin{aligned}
M_{1} M_{2} & \leq c^{2} D^{4} a_{1} a_{2} \log ^{2} B \\
\max \left(M_{1}, M_{2}\right) & \leq c D^{2} \max \left(a_{1}, a_{2}\right) \log B \\
\min \left(M_{1}, M_{2}\right) & \leq c D^{2} \min \left(a_{1}, a_{2}\right) \log B \\
c D^{2} \min \left(a_{1}, a_{2}\right) \log B & \geq 4 c \log ^{2} p>e
\end{aligned}
$$


et

$$
\log \left(c D^{2} \min \left(a_{1}, a_{2}\right) \log B\right) \geq \frac{\log \left(4 c \log ^{2} p\right.}{4 \log ^{2} p} D^{2} \min \left(a_{1}, a_{2}\right) \log B
$$

d'où

$$
\begin{aligned}
- & \log \Delta \leq \\
\leq & 4\left(\frac{1}{p-1}+\frac{1}{p^{2}}\right) c^{2} \log p \cdot D^{4} a_{1} a_{2} \log ^{2} B+ \\
& +2 c D^{2} \max \left(a_{1}, a_{2}\right) \log B \times \\
& \times\left\{\left[\frac{1}{\log p}+\frac{\log \left(4 c \log ^{2} p\right)}{4 \log ^{2} p}\right] D^{2} \min \left(a_{1}, a_{2}\right) \log B+2 \log 2+\frac{\log p}{p-1}\right\} \\
\leq & \left\{4\left(\frac{1}{p-1}+\frac{1}{p^{2}}\right) c^{2} \log p+2\left[\frac{1}{\log p}+\frac{\log \left(4 c \log ^{2} p\right)}{4 \log ^{2} p}\right] c\right\} D^{4} a_{1} a_{2} \log ^{2} B \\
& +2\left(\log 4+\frac{\log p}{p-1}\right) c D^{2}\left(a_{1}+a_{2}\right) \log B . \square
\end{aligned}
$$

Il existe une base de $\mathbb{K}_{0}=\mathbb{Q}\left(\alpha_{1}, \alpha_{2}\right)$ de la forme $\xi_{d}=\alpha_{1}^{d_{1}} \alpha_{2}^{d_{2}}, 0 \leq d_{i}<D_{i}$, $i=1,2, d_{1}+d_{2} \leq D_{0}, d=1,2, \ldots, D_{0}$ où $D_{i}=\operatorname{deg}\left(\alpha_{i}\right)=\left[\overline{\mathbb{Q}}\left(\alpha_{i}\right): \mathbb{Q}\right]$, $i=1,2$.

Supposons dans un premier temps que $p \geq 3$.

Cas 4.

$$
\begin{aligned}
\text { les points } \alpha_{1}^{p u} \alpha_{2}^{p v},-U_{1} \leq u \leq U_{1},-V_{1} \leq v \leq V_{1} \\
\text { sont deux à deux distincts. }
\end{aligned}
$$

les points $u+v \beta,-U_{2} \leq u \leq U_{2},-V_{2} \leq v \leq V_{2}$ sont deux à deux distincts.

$$
B c\left(a_{1}+a_{2}\right) \geq\left(c_{0} / 2\right) D a_{1} a_{2} .
$$

Pour $(u, v) \in \mathbb{Z}^{2}$, on pose

$$
\varphi(u, v)=\sum_{h=0}^{L_{0}} \sum_{k=-L_{1}}^{L_{1}} p_{h k} \Delta_{h}\left(b_{2} u+b_{1} v\right) \alpha_{1}^{p k u} \alpha_{2}^{p k v}
$$

où

$$
\begin{aligned}
& p_{h k}=\sum_{d=1}^{D_{0}} p_{h k d} \xi_{d}, \quad 0 \leq h \leq L_{0},-L_{1} \leq k \leq L_{1} \\
& p_{h k d} \in \mathbb{Z}, \quad 0 \leq h \leq L_{0},-L_{1} \leq k \leq L_{1}, d=1,2, \ldots, D_{0} .
\end{aligned}
$$




\section{Dong Pingping}

Proposition 4.5. - Il existe $p_{h k d} \in \mathbb{Z}$ non tous nuls tels que

$$
\varphi(u, v)=0, \quad-M_{1} \leq u \leq M_{1},-M_{2} \leq v \leq M_{2}
$$

avec

$$
\begin{aligned}
\log \max \left\|p_{h k d}\right\| \leq & \eta\left\{c_{2} D^{3} a_{1} a_{2} \log ^{2} B-c_{0} \log p \cdot D^{3} a_{1} a_{2} \log ^{2} B+\right. \\
& \left.+c_{3} D^{2}\left(a_{1}+a_{2}\right) \log B\right\}
\end{aligned}
$$

où

$$
\begin{aligned}
& c_{2}=c_{0}+2 p c c_{1} \\
& c_{3}=p\left(c+\frac{c_{1}}{2}\right)+\frac{1}{8 \log ^{2} p}\left[\log \left(4 c_{1} \log p+1\right)+\frac{5}{2} \log 2+\log \zeta\right] .
\end{aligned}
$$

Démonstration. - On utilise le lemme 2.1 avec " $\mu$ ", " $\nu$ " indiqués dans (23), on déduit facilement de (21), (24), (25) que $\nu>D_{0} \mu$ et

$$
\begin{gathered}
\frac{D_{0} \mu}{\nu-D_{0} \mu} \leq \eta . \\
\varphi(u, v)=\sum_{h=0}^{L_{0}} \sum_{k=L_{1}}^{L_{1}} \sum_{d=1}^{D_{0}} p_{h k d} \Delta_{h}\left(b_{2} u+b_{1} v\right) \alpha_{1}^{p k u+d_{1}} \alpha_{2}^{p k v+d_{2}} ;
\end{gathered}
$$

pour $(u, v) \in \mathbb{Z}^{2}$, les coefficients de $\varphi(u, v)$ sont des polynômes en $\alpha_{1}, \alpha_{2}$, $\alpha_{1}^{-1}, \alpha_{2}^{-1}$ à coefficients entiers dans les degrés par rapport à $\alpha_{1}, \alpha_{2}, \alpha_{1}^{-1}$, $\alpha_{2}^{-1}$ sont inférieurs ou égaux à

$$
\begin{array}{ll}
N_{1}(u, v)=p L_{1}\|u\|+D-1 & N_{2}(u, v)=p L_{1}\|v\|+D-1 \\
N_{1}^{\prime}(u, v)=p L_{1}\|u\| & N_{2}^{\prime}(u, v)=p L_{1}\|v\|
\end{array}
$$

respectivement.

Pour $(u, v) \in \mathbb{Z}^{2},-M_{1} \leq u \leq M_{1},-M_{2} \leq v \leq M_{2}$ et pour $0 \leq h \leq L_{0}$, on a, grâce à (33)

$$
\begin{aligned}
\max \left(\left\|b_{2} u+b_{1} v\right\|, \frac{h}{2}\right) & \leq \max \left(B\left(M_{1}+M_{2}\right), \frac{L_{0}}{2}\right) \\
& \leq \max \left\{B c\left(a_{1}+a_{2}\right) D^{2} \log B, \frac{c_{0}}{2} D^{3} a_{1} a_{2} \log B\right\} \\
& =E
\end{aligned}
$$


Minoration de combinaisons linéaires de deux logarithmes $p$-adiques

où on a posé $E=B c\left(a_{1}+a_{2}\right) D^{2} \log B$ et on a

$$
\left\|\Delta_{h}\left(b_{2} u+b_{1} v\right)\right\| \leq 2 \frac{E^{h}}{h !} .
$$

La somme $L(u, v)$ des longueurs de tous les coefficients de $\varphi(u, v)$ vérifie

$$
\begin{aligned}
L(u, v) & \leq D\left(2 L_{1}+1\right) \sum_{h=0}^{L_{0}}\left\|\Delta_{h}\left(b_{2} u+b_{1} v\right)\right\| \leq 2 D\left(2 L_{1}+1\right) \sum_{h=0}^{L_{0}} \frac{E^{h}}{h !} \\
& \leq 2 D\left(2 L_{1}+1\right)\left(\frac{B}{p}\right)^{L_{0}} \sum_{h=0}^{L_{0}} \frac{1}{h !}\left(p c\left(a_{1}+a_{2}\right) D^{2} \log B\right)^{h} \leq L
\end{aligned}
$$

avec

$$
L=2 D\left(2 L_{1}+1\right)\left(\frac{B}{p}\right)^{c_{0} D^{3} a_{1} a_{2} \log B} B^{p c\left(a_{1}+a_{2}\right) D^{2}}
$$

donc

$$
\begin{aligned}
V(u, v) & =L(u, v) e^{N_{1}(u, v) h\left(\alpha_{1}\right)+N_{2}(u, v) h\left(\alpha_{2}\right)+N_{1}^{\prime}(u, v) h\left(\alpha_{1}^{-1}\right)+N_{2}^{\prime}(u, v) h\left(\alpha_{2}^{-1}\right)} \\
& \leq L e^{2 p L_{1}\left(a_{1}\|u\|+a_{2}\|v\|\right)+(D-1)\left(a_{1}+a_{2}\right)}
\end{aligned}
$$

Alors, le lemme 2.1 dit que le système linéaire (34) possède une solution

$$
p_{h k d} \in \mathbb{Z}, \quad 0 \leq h \leq L_{0},-L_{1} \leq k \leq L_{1}, d=1,2, \ldots, D_{0}
$$

non nulle vérifiant

$$
\begin{aligned}
& \log \max \left\|p_{h k d}\right\| \leq \frac{1}{\nu-D_{0} \mu}\left\{\mu \log 2+D_{0} \sum_{u=-M_{1}}^{M_{1}} \sum_{v=-M_{2}}^{M_{2}} \log V(u, v)\right\} \quad(36) \\
& \leq \frac{1}{\nu-D_{0} \mu}\left\{\mu \log 2+D_{0}\left[\mu \log L+2 p L_{1} \times\right.\right. \\
&\left.\left.\times \sum_{u=-M_{1}}^{M_{1}} \sum_{v=-M_{2}}^{M_{2}}\left(a_{1}\|u\|+a_{2}\|v\|\right)+\mu(D-1)\left(a_{1}+a_{2}\right)\right]\right\} \\
&= \frac{1}{\nu-D_{0} \mu}\left\{\mu \log 2+D_{0}[\mu \log L+\right. \\
&+2 p L_{1}\left(M_{1}\left(M_{1}+1\right)\left(2 M_{2}+1\right) a_{1}+\right. \\
&-221-
\end{aligned}
$$




$$
\begin{aligned}
& \left.\left.\left.+M_{2}\left(M_{2}+1\right)\left(2 M_{2}+1\right) a_{2}\right)+\mu(D-1)\left(a_{1}+a_{2}\right)\right]\right\} \\
\leq & \frac{D_{0} \mu}{\nu-D_{0} \mu}\{\log 2+\log L+ \\
& \left.+p L_{1}\left[\left(M_{1}+\frac{1}{2}\right) a_{1}+\left(M_{2}+\frac{1}{2}\right) a_{2}\right](D-1)\left(a_{1}+a_{2}\right)\right\} \\
\leq & \eta\left\{\log L+p L_{1}\left(M_{1} a_{1}+M_{2} a_{2}\right)+\right. \\
& \left.+\log 2+\left(\frac{p L_{1}}{2}+D-1\right)\left(a_{1}+a_{2}\right)\right\} .
\end{aligned}
$$

Mais $\zeta \log B \geq 2 \zeta \log p \geq e ;$ alors

$$
\log \log B \leq \log (\zeta \log B) \leq \frac{\log (2 \zeta \log p)}{2 \log p} \log B
$$

$$
\begin{aligned}
\log 2 D\left(2 L_{1}+1\right) \leq & \log \left[2\left(2 c_{1}+\frac{1}{2 \log p}\right) D^{2} \log B\right] \\
\leq & \log \left[2\left(2 c_{1}+\frac{1}{2 \log p}\right)\right]+ \\
& +\frac{\log 2}{2} D^{2}+\frac{\log (2 \zeta \log p)}{2 \log p} \log B \\
\leq & c_{4} D^{2}\left(a_{1}+a_{2}\right) \log B
\end{aligned}
$$

avec

$$
c_{4}=\frac{1}{8 \log ^{2} p}\left[\log \left(4 c_{1} \log p+1\right)+\frac{3}{2} \log 2+\log \zeta\right] .
$$

On a aussi

$$
\begin{aligned}
& \left\{\begin{array}{l}
L_{1}\left(M_{1} a_{1}+M_{2} a_{2}\right) \leq 2 c c_{1} D^{3} a_{1} a_{2} \log ^{2} B \\
\log 2+\left(\frac{p L_{1}}{2}+D-1\right)\left(a_{1}+a_{2}\right) \leq \log 2+ \\
\quad+\left(\frac{p c_{1}}{2} D \log B+D-1\right)\left(a_{1}+a_{2}\right)
\end{array}\right. \\
& \leq\left(\frac{p c_{1}}{2 D} \log B+\frac{D-1}{D^{2}}+\frac{\log 2}{4 \log p}\right) D^{2}\left(a_{1}+a_{2}\right) \\
& \leq\left(\frac{p c_{1}}{2 D}+\frac{\log 2}{8 \log ^{2} p}\right) D^{2}\left(a_{1}+a_{2}\right) \log B \text {. }
\end{aligned}
$$

On déduit (35) de (36), (37) et (38). 
On voit facilement que $p_{h k}, 0 \leq h \leq L_{0},-L_{1} \leq k \leq L$ ne sont pas tous nuls et que

$$
\left|p_{h k}\right| \leq 1, \quad 0 \leq h \leq L_{0},-L_{1} \leq k \leq L_{1}
$$

On pose

$$
F(z)=\sum_{h=0}^{L_{0}} \sum_{k=-L_{1}}^{L_{1}} p_{h k} \Delta_{h}\left(b_{2} z\right) \alpha_{1}^{p k z}, \quad z \in \mathbb{C}_{p}, \nu(z) \geq-1
$$

de sorte que

$$
F(u+v \beta)=\sum_{h=0}^{L_{0}} \sum_{k=-L_{1}}^{L_{1}} p_{h k} \Delta_{h}\left(b_{2} u+b_{1} v\right) \alpha_{1}^{p k u} \alpha_{1}^{p k \beta v}
$$

On a donc

$$
\varphi(u, v)-F(u+v \beta)=\sum_{h=0}^{L_{0}} \sum_{k=-L_{1}}^{L_{1}} p_{h k} \Delta_{h}\left(b_{2} u+b_{1} v\right) \alpha_{1}^{p k u}\left(\alpha_{2}^{p k u}-\alpha_{1}^{p k \beta v}\right)
$$

on utilise le lemme 2.5 pour trouver

$$
\begin{aligned}
|\varphi(u, v)-F(u+v \beta)| & \leq \max _{-L_{1} \leq k \leq L_{1}}\left|\alpha_{2}^{p k v}-\alpha_{1}^{p k \beta v}\right|=\max _{-L_{1} \leq k \leq L_{1}}\left|\frac{p k v}{b_{2}}\right||\Lambda| \\
& \leq \frac{|\Lambda|}{p}
\end{aligned}
$$

pour tout $(u, v) \in \mathbb{Z}^{2}$.

Proposition 4.6. - Sous l'hypothèse (H), on a

$$
\log |\varphi(u, v)| \leq \max \left\{\log |F|_{p}-\mu \log p, \log \frac{|\Lambda|}{p}-\log \Delta\right\}
$$

pour tout $(u, v) \in \mathbb{Z}^{2}$ et

$$
\begin{aligned}
\log |F|_{p}-\mu \log p< & {\left[\left(2 c-\frac{2}{4 \log ^{2} p}\right)^{2} D^{4} a_{1} a_{2} \log ^{2} B+\right.} \\
& \left.-\frac{p}{p-1} c_{0} D^{3} a_{1} a_{2} \log B\right] \log p \\
& -223-
\end{aligned}
$$




\section{Dong Pingping}

Démonstration. - La fonction $F$ est analytique sur le disque $|z| \leq p$ et vérifie

$$
|F|_{p} \leq \max _{0 \leq h \leq L_{0}}\left|\Delta_{h}\left(b_{2} z\right)\right|_{p}=\max _{0 \leq h \leq L_{0}}\left|\Delta_{h}(z)\right|_{p}<p^{p_{L_{0}} /(p-1)} .
$$

Pour $\gamma=u^{\prime}+v^{\prime} \beta \in \Gamma,\left\|u^{\prime}\right\| \leq M_{1},\left\|v^{\prime}\right\| \leq M_{2}$, on a $\varphi\left(u^{\prime}, v^{\prime}\right)=0$, donc

$$
|F(\gamma)|=\left|F(\gamma)-\varphi\left(u^{\prime}, v^{\prime}\right)\right| \leq \frac{|\Lambda|}{p} .
$$

On utilise le lemme 2.3 avec $R=p, r=1,\left\{z_{1}, \ldots, z_{s}\right\}=\Gamma, s=|\Gamma|=\mu$

$$
\begin{aligned}
|F|_{1} & \leq \max \left\{|F|_{p} p^{-\mu}, \frac{|F(\gamma)|}{\Delta}, \gamma \in \Gamma\right\} \\
& \leq \max \left\{|F|_{p} p^{-\mu}, \frac{|\Lambda|}{p \Delta}\right\} .
\end{aligned}
$$

Pour $(u, v) \in \mathbb{Z}^{2}$, on a

$$
\begin{aligned}
|\varphi(u, v)| & \leq \max (|\varphi(u, v)-F(u+v \beta)|,|F(u+v \beta)|) \\
& \leq \max \left(\frac{|\Lambda|}{p},|F|_{1}\right) \leq \max \left(|F|_{p} p^{-\mu}, \frac{|\Lambda|}{p \Delta}\right) .
\end{aligned}
$$

On a donc (40); et

$$
\begin{aligned}
\log |F|_{p}-\mu \log p< & \frac{p L_{0}}{p-1} \log p-\mu \log p \\
\leq & -\left[\left(2 c-\frac{1}{4 \log ^{2} p}\right)^{2} D^{4} a_{1} a_{2} \log ^{2} B+\right. \\
& \left.-\frac{p}{p-1} c_{0} D^{3} a_{1} a_{2} \log B\right] \log p .
\end{aligned}
$$

Proposition 4.7. - On pose $(u, v) \in \mathbb{Z}^{2},\|u\| \leq M_{1}^{*},\|v\| \leq M_{2}^{*}$. Alors ou bien $\varphi(u, v)=0$, ou bien $\log |\varphi(u, v)| \geq-S_{1}$, où l'on a posé

$S_{1}=c_{5} D^{4} a_{1} a_{2} \log ^{2} B-(\eta+1) c_{0} \log p D^{4} a_{1} a_{2} \log B+c_{6} D^{3}\left(a_{1}+a_{2}\right) \log B$ 
Minoration de combinaisons linéaires de deux logarithmes $p$-adiques

avec

$$
\begin{aligned}
c_{5}= & (1+\eta) c_{0}+2 p(\eta+2 \chi) c c_{1} \\
c_{6}= & p\left[(\eta+\chi) c+\eta \frac{c_{1}}{2}\right]+ \\
& +\frac{\eta+1}{8 \log ^{2} p}\left[\log \left(4 c_{1} \log p+1\right)+\frac{3}{2} \log 2+\log \zeta\right]+\frac{\eta \log 2}{8 \log ^{2} p}+\frac{1}{8 \log p} .
\end{aligned}
$$

Démonstration. - Nous utilisons le lemme 2.4 (b) :

$$
\varphi(u, v)=\sum_{h=0}^{L_{0}} \sum_{k=-L_{1}}^{L_{1}} \sum_{d=1}^{D_{0}} p_{h k d} \Delta_{h}\left(b_{2} u+b_{1} v\right) \alpha_{1}^{p k u+d_{1}} \alpha_{2}^{p k v+d_{2}}
$$

est un polynôme en $\alpha_{1}, \alpha_{2}, \alpha_{1}^{-1}, \alpha_{2}^{-1}$ à coefficients entiers. Soient $N_{1}, N_{2}$, $N_{1}^{\prime}, N_{2}^{\prime}$ les degrés de $\varphi$ par rapport à $\alpha_{1}, \alpha_{2}, \alpha_{1}^{-1}, \alpha_{2}^{-1}$ respectivement. On voit que :

$$
\begin{array}{ll}
N_{1} \leq p L_{1} M_{1}^{*}+D-1 & N_{2} \leq p L_{1} M_{2}^{*}+D-1 \\
N_{1}^{\prime} \leq p L_{1} M_{1}^{*} & N_{2}^{\prime} \leq p L_{1} M_{2}^{*} .
\end{array}
$$

De plus le nombre $S=N_{1} h\left(\alpha_{1}\right)+N_{2} h\left(\alpha_{2}\right)+N_{1}^{\prime} h\left(\alpha_{1}^{-1}\right)+N_{2}^{\prime} h\left(\alpha_{2}^{-1}\right)$ est majoré par

$$
\begin{aligned}
& \left(N_{1}+N_{1}^{\prime}\right) a_{1}+\left(N_{2}+N_{2}^{\prime}\right) a_{2} \leq \\
& \quad \leq 2 p L_{1}\left(M_{1}^{*} a_{1}+M_{2}^{*} a_{2}\right)+(D-1)\left(a_{1}+a_{2}\right) \\
& \quad \leq 4 p \chi c c_{1} a_{1} a_{2} D^{3} \log ^{2} B+\frac{1}{8 \log p} D^{2}\left(a_{1}+a_{2}\right) \log B .
\end{aligned}
$$

Pour $0 \leq h \leq L_{0}$, on a grâce à (33)

$$
\begin{aligned}
\max \left(\left\|b_{2} u+b_{1} v\right\|, \frac{h}{2}\right) & \leq \max \left(B\left(M_{1}^{*}+M_{2}^{*}\right), \frac{L_{0}}{2}\right) \\
& \leq \max \left(B c \chi\left(a_{1}+\alpha_{2}\right) D^{2} \log B, \frac{c_{0}}{2} D^{3} a_{1} a_{2} \log B\right) \\
& =\chi E
\end{aligned}
$$

et

$$
\left\|\Delta_{h}\left(b_{2} u+b_{1} v\right)\right\| \leq 2 \frac{(\chi E)^{h}}{h !}
$$




\section{Dong Pingping}

Ainsi, la longueur de $\varphi$ vérifie

$$
\begin{aligned}
L(\varphi) & \leq \sum_{h=0}^{L_{0}} \sum_{k=-L_{1}}^{L_{1}} \sum_{d=1}^{D_{0}}\left\|p_{h k d}\right\|\left\|\Delta_{h}\left(b_{2} u+b_{1} v\right)\right\| \\
& \leq 2 D\left(2 L_{1}+1\right) \max \left\|p_{h k d}\right\| \sum_{h=0}^{L_{0}} \frac{(\chi E)^{h}}{h !} \\
& \leq 2 D\left(2 L_{1}+1\right) \max \left\|p_{h k d}\right\|\left(\frac{B}{p}\right)^{L_{0}} \sum_{h=0}^{L_{0}} \frac{\left(p \chi c\left(a_{1}+a_{2}\right) D^{2} \log B\right)^{h}}{h !} \\
& \leq 2 D\left(2 L_{1}+1\right) \max \left\|p_{h k d}\right\|\left(\frac{B}{p}\right)^{c_{0} D^{3} a_{1} a_{2} \log B} B^{p \chi c\left(a_{1}+a_{2}\right) D^{2}} .
\end{aligned}
$$

On utilise (37) et la proposition 4.5

$$
\begin{aligned}
\log L(\varphi) \leq & c 4 D^{2}\left(a_{1}+a_{2}\right) \log B+\eta\left\{c_{4} D^{3} a_{1} a_{2} \log ^{2} B+\right. \\
& \left.-c_{0} \log p \cdot D^{3} a_{1} a_{2} \log B+c_{3} D^{2}\left(a_{1}+a_{2}\right) \log B\right\}+ \\
& +c_{0} D^{3} a_{1} a_{2} \log ^{2} B-c_{0} \log p \cdot D^{3} a_{1} a_{2} \log B+ \\
& +p \chi c D^{2}\left(a_{1}+a_{2}\right) \log B \\
\leq & \left(c_{0}+\eta c_{2}\right) D^{3} a_{1} a_{2} \log B-(\eta+1) c_{0} \log p \cdot D^{3} a_{1} a_{2} \log B+ \\
& +\left(c_{4}+\eta c_{3}+p c \chi\right) D^{2}\left(a_{1}+a_{2}\right) \log B .
\end{aligned}
$$

Si $\varphi(u, v) \neq 0$, alors

$$
\begin{aligned}
\log |\varphi(u, v)| \geq & -D(\log L(\varphi)+S) \\
\geq & -\left(c_{0}+\eta c_{2}+4 p \chi c c_{1}\right) D^{4} a_{1} a_{2} \log ^{2} B+ \\
& +(\eta+1) c_{0} \log p \cdot D^{4} a_{1} a_{2} \log B+ \\
& -\left(c_{4}+\eta c_{3}+p \chi c+\frac{1}{8 \log p}\right) D^{3}\left(a_{1}+a_{2}\right) \log B
\end{aligned}
$$

mais

$$
c_{5}=c_{0}+\eta c_{2}+4 p \chi c c_{1}, \quad c_{6}=c_{4}+\eta c_{3}+p \chi c+\frac{1}{8 \log p} .
$$

Nous imposons ici deux nouvelles restrictions sur $c, c_{0}, c_{1}$ et $\chi$.

$$
\begin{aligned}
& \left(2 c-\frac{1}{4 \log ^{2} p}\right)^{2} \log p \geq c_{5} \\
& \left(\eta-\frac{1}{p-1}\right) c_{0} \log ^{2} p \geq c_{6} .
\end{aligned}
$$


On pose

$$
P(X, Y)=\sum_{h=0}^{L_{0}} \sum_{k=-L_{1}}^{L_{1}} p_{h k} \Delta_{h}\left(b_{2} X\right) Y^{L_{1}+k} \in \mathbb{C}[X, Y] .
$$

On déduit que $P \neq 0$ du fait que les coefficients $p_{h k}, 0 \leq h \leq L_{0}$, $-L_{1} \leq k \leq L_{1}$ ne sont pas tous nuls.

Le polynôme $P$ est de degré au plus $L_{0}$ et $2 L_{1}$ par rapport à $X$ et $Y$ respectivement.

L'hypothèse (21) implique $U_{2} \geq U_{1}, V_{2} \geq V_{1}$, et (32) implique que les points $u+v \beta,-U_{1} \leq u \leq U_{1},-V_{1} \leq v \leq V_{1}$ sont deux à deux distincts.

La première partie de (21) implique

$$
\begin{aligned}
\left(2 \chi_{1} c\right. & \left.-\frac{1}{4 \log ^{2} p}\right)^{2} 2 \log p>c_{0} \geq \frac{c_{1}}{2 \log ^{2} p} \\
\left(2 U_{1}+1\right)\left(2 V_{1}+1\right) & \geq\left(2 \chi_{1} c-\frac{1}{4 \log ^{2} p}\right)^{2} D^{4} a_{1} a_{2} \log ^{2} B \\
& \geq\left(2 \chi_{1} c-\frac{1}{4 \log ^{2} p}\right)^{2} 2 \log p \cdot D^{3} a_{1} a_{2} \log B \\
& >c_{0} D^{3} a_{1} a_{2} \log B \\
& \geq\left\{\begin{array}{l}
\frac{L_{0} c_{1}}{2 \log ^{2} p} D^{3} a_{1} a_{2} \log B \geq 2 c_{1} D \log B \geq 2 L_{1} .
\end{array}\right.
\end{aligned}
$$

La deuxième partie de (21) implique

$$
\begin{aligned}
&\left(2 \chi_{2} c-\frac{1}{4 \log ^{2} p}\right)^{2}>4 c_{0} c_{1} \\
&\left(2 U_{2}+1\right)\left(2 V_{2}+1\right) \geq\left(2 \chi_{2} c-\frac{1}{4 \log ^{2} p}\right)^{2} D^{4} a_{1} a_{2} \log ^{2} B \\
&>4 c_{0} c_{1} D^{4} a_{1} a_{2} \log ^{2} B \geq 2 L_{0} \cdot 2 L_{1} .
\end{aligned}
$$

On voit aussi que

$$
\begin{aligned}
M_{1}^{*} & \geq\left(\chi-\frac{1}{4 c \log ^{2} p}\right) c D^{2} a_{2} \log B \\
& \geq\left(\chi_{1}+\chi_{2}\right) c D^{2} a_{2} \log ^{2} B \geq U_{1}+U_{2} \\
M_{2}^{*} & \geq\left(\chi-\frac{1}{4 c \log ^{2} p}\right) c D^{2} a_{1} \log B \\
& \geq\left(\chi_{1}+\chi_{2}\right) c D^{2} a_{1} \log ^{2} B \geq V_{1}+V_{2}
\end{aligned}
$$




\section{Dong Pingping}

En utilisant (31), (32), il résulte du lemme 2.6 qu'il existe un point $\left(u_{0}, v_{0}\right) \in \mathbb{Z}^{2} .\left\|u_{0}\right\| \leq M_{1}^{*},\left\|v_{0}\right\| \leq M_{2}^{*}$ tel que $P\left(u_{0}+v_{0} \beta, \alpha_{1}^{p u_{0}} \alpha_{2}^{p v_{0}}\right) \neq 0$, ce qui équivaut à $\varphi\left(u_{0}, v_{0}\right) \neq 0$. Puisque $\chi_{2}>1, U_{2} \geq M_{1}, V_{2} \geq M_{2}$, (32) implique l'hypothèse $(\mathrm{H})$. Alors, on utilise les propositions 4.6 et 4.7 , avec $(u, v)=\left(u_{0}, v_{0}\right)$

$$
\max \left\{\log |F|_{p}-\mu \log p, \log \frac{|\Lambda|}{p}-\log \Delta\right\} \geq \log \left|\varphi\left(u_{0}, v_{0}\right)\right| \geq-S_{1} ;
$$

mais (43) implique

$$
\frac{p}{p-1} c_{0} \log p \leq(\eta+1) c_{0} \log p-\frac{c_{6}}{\log p} ;
$$

alors par (41), (42) et (44), on a

$$
\begin{aligned}
\log |F|_{p}-\mu \log p< \\
<-\left\{\left(2 c-\frac{1}{4 \log ^{2} p}\right)^{2} D^{4} a_{1} a_{2} \log ^{2} B-\frac{p}{p-1} c_{0} D^{3} a_{1} a_{2} \log B\right\} \log p \\
\leq-\left\{c_{5} D^{4} a_{1} a_{2} \log ^{2} B-\left[(\eta+1) c_{0} \log p-\frac{c_{6}}{\log p}\right] D^{4} a_{1} a_{2} \log B\right\} \\
\leq-S_{1}
\end{aligned}
$$

donc

$$
\log \frac{|\Lambda|}{p}-\log \Delta \geq-S_{1}
$$

et on utilise la proposition 4.4

$$
\begin{aligned}
\log \frac{|\Lambda|}{p} \geq & \log \Delta-S_{1} \\
\geq & -\left\{4\left(\frac{1}{p-1}+\frac{1}{p^{2}}\right) c_{2} \log p+c_{5}+\right. \\
& \left.+2\left[\frac{1}{\log p}+\frac{\log \left(4 c \log ^{2} p\right)}{4 \log ^{2} p}\right] c\right\} D^{4} a_{1} a_{2} \log ^{2} B+ \\
+ & (\eta+1) c_{0} \log p \cdot D^{4} a_{1} a_{2} \log B+ \\
& -\left[c_{6}+2\left(\log 4+\frac{\log p}{p-1}\right) c\right] D^{3}\left(a_{1}+a_{2}\right) \log B .
\end{aligned}
$$

Maintenant nous nous mettons à choisir $c, c_{0}, c_{1}$ et puis $\chi_{1}, \chi_{2}, \chi$. 
Minoration de combinaisons linéaires de deux logarithmes $p$-adiques

Soient $t_{0}, t_{1}, \lambda_{0}$ des constantes absolues, $\lambda$ un nombre réel ne dépendant que de $p$ et satisfaisant les conditions suivantes

$$
\begin{aligned}
& t_{1} \geq \frac{2}{3}, \quad 3 t_{1} \leq t_{0}, \quad \lambda>\lambda_{0} \geq 2 \\
& w=t_{0} t_{1} \geq 3
\end{aligned}
$$

et

$$
\begin{aligned}
4 \lambda\left(1-\frac{1}{8 \lambda_{0}^{2} p}\right)^{2} \geq \tau_{1}= & \left(\frac{2}{w Z_{0}-2}+1\right) t_{0}+ \\
& +4\left(\frac{1}{w Z_{0}-2}+\sqrt{w}+\frac{1}{2} \sqrt{\frac{t_{0}}{2 \lambda}}+\frac{1}{2 \lambda_{0}^{2} p}\right) t_{1},
\end{aligned}
$$

où $Z_{0}=\left(1-\left(1 / 4 t_{1} \lambda\right)\right)\left(1-\left(1 / 4 \lambda_{0}^{2} p\right)\right)$,

$$
\begin{gathered}
\tau=p^{2} \lambda\left[t_{0} \lambda^{2} \log p-\left(\lambda+t_{1} \frac{\log p}{2 p}\right)\right]+ \\
-\frac{1}{8}\left[\log \left(4 t_{1} \lambda+1\right)+\frac{5}{2} \log 2+\log \zeta\right] \geq 0 \\
\left(\frac{2}{w\left(1-\frac{1}{4 t_{1} \lambda}\right)-2}-\frac{1}{p-1}\right) t_{0} \lambda^{3} p^{2} \geq \\
\geq \frac{1}{\log p}\left\{p^{2} \lambda^{2}\left(\frac{2}{w\left(1-\frac{1}{4 t_{1} \lambda}\right)-2}+\sqrt{w}+\frac{1}{2} \sqrt{\frac{t_{0}}{2 \lambda}}+\frac{1}{2 \lambda_{0}^{2} p}\right)+\right. \\
+\left(\frac{2}{w\left(1-\frac{1}{4 t_{1} \lambda}\right)-2}+1\right) \frac{1}{8}\left[\log \left(4 t_{1} \lambda+1\right)+\frac{3}{2} \log 2+\log \zeta\right]+ \\
\left.+\frac{1}{w\left(1-\frac{1}{4 t_{1} \lambda}\right)-2} \frac{\log 2}{4}\right\}+\frac{t_{1} \lambda p}{w\left(1-\frac{1}{4 t_{1} \lambda}\right)-2}+\frac{1}{8}
\end{gathered}
$$

\section{Choisissons}

$$
\begin{aligned}
c_{1}=\frac{t_{1} \lambda}{\log p}, \quad c & =\frac{\lambda^{2} p}{\log ^{2} p}, \quad c_{0}=\frac{t_{0} \lambda^{3} p^{2}}{\log ^{3} p} . \\
& -229-
\end{aligned}
$$




\section{Dong Pingping}

(46) signifie que $c \geq 2, c_{0} \geq 1, c_{1} \geq 1 / \log p$ et $2 \log ^{2} p \cdot c_{0} \geq c_{1}$ et on déduit aussi de $t_{1} \geq 2 / 3, \lambda_{0} \geq 2$ et (47) que

$$
\begin{aligned}
Z & =c_{0}\left(2 c_{1}-\frac{1}{2 \log p}\right)\left(2 c+\frac{1}{4 \log ^{2} p}\right)^{-2} \\
& =\frac{c_{0} c_{1}}{2 c^{2}}\left(1-\frac{1}{4 c_{1} \log p}\right)\left(1+\frac{1}{8 c \log ^{2} p}\right)^{-2} \\
& =\frac{w}{2}\left(1-\frac{1}{4 t_{1} \lambda}\right)\left(1+\frac{1}{8 \lambda^{2} p}\right)^{-2}>\frac{w}{2} Z_{0}>1
\end{aligned}
$$

Alors (19), (20) sont vérifiées. On voit que $\eta=1 /(Z-1)$, donc

$$
\frac{2}{w\left(1-\frac{1}{4 t_{1} \lambda}\right)-2}<\eta<\frac{2}{w Z_{0}-2}
$$

Choisissons de plus

$$
\begin{aligned}
\chi_{1} & =\frac{1}{2} \sqrt{\frac{t_{0}}{2 \lambda}}+\frac{1}{8 \lambda_{0}^{2} p}, \quad \chi_{2}=\sqrt{w}+\frac{1}{8 \lambda_{0}^{2} p} \\
\chi & =\sqrt{w}+\frac{1}{2} \sqrt{\frac{t_{0}}{2 \lambda}}+\frac{1}{2 \lambda_{0}^{2} p} .
\end{aligned}
$$

En utilisant (46), (47) les relations (20), (21) deviennent claires.

On utilise (51), (52) et puis (48) pour obtenir

$$
\left(2 c-\frac{1}{4 \log ^{2} p}\right)^{2} \log p \geq \frac{4 \lambda^{4} p^{2}}{\log ^{4} p}\left(1-\frac{1}{8 \lambda_{0}^{2} p}\right)^{2} \log p \geq \tau_{1} \frac{\lambda^{3} p^{2}}{\log ^{3} p} \geq c_{5},
$$

c'est (42), et

$$
\begin{gathered}
c_{0} \log ^{2} p-p\left(c+\frac{c_{1}}{2}\right)+ \\
-\frac{1}{8 \log ^{2} p}\left[\log \left(4 c_{1} \log p+1\right)+\frac{5}{2} \log 2+\log \zeta\right]=\frac{\tau}{\log ^{2} p} \\
-230-
\end{gathered}
$$


La condition (50) peut se transformer en

$$
\begin{aligned}
& \left(\frac{2}{w\left(1-\frac{1}{4 t_{1} \lambda}\right)-2}-\frac{1}{p-1}\right) c_{0} \log ^{2} p \geq \\
& \geq p c\left(\frac{2}{w\left(1-\frac{1}{4 t_{1} \lambda}\right)-2}+\chi\right)+\left(\frac{2}{w\left(1-\frac{1}{4 t_{1} \lambda}\right)-2}+1\right) \times \\
& \quad \times \frac{1}{8 \log ^{2} p}\left[\log \left(4 c_{1} \log p+1\right)+\frac{3}{2} \log 2+\log \zeta\right]+ \\
& +\frac{2}{w\left(1-\frac{1}{4 t_{1} \lambda}\right)-2} \frac{\log 2}{8 \log ^{2} p}+\frac{2}{w\left(1-\frac{1}{4 t_{1} \lambda}\right)-2} \frac{p c_{1}}{2}+\frac{1}{8 \log p},
\end{aligned}
$$

ce qui équivaut à

$$
\begin{aligned}
& \frac{2}{w\left(1-\frac{1}{4 t_{1} \lambda}\right)-2} \frac{\tau}{\log ^{2} p} \leq \\
& \leq \frac{1}{p-1} c_{0} \log ^{2}+p \chi c+\frac{1}{8 \log ^{2} p}\left[\log \left(4 c_{1} \log p+1\right)+\frac{3}{2} \log 2+\log (\zeta p)\right] .
\end{aligned}
$$

Alors par (51), on a

$$
\begin{aligned}
\eta \frac{\tau}{\log ^{2} p} \geq & \frac{1}{p-1} c_{0} \log ^{2} p+p \chi c+ \\
& +\frac{1}{8 \log ^{2} p}\left[\log \left(4 c_{1} \log p+1\right)+\frac{3}{2} \log 2+\log (\zeta p)\right]
\end{aligned}
$$

ce qui équivaut à (43).

Choisissons $t_{0}=4.4, t_{1}=0.95, \lambda_{0}=5$ et $\lambda=\lambda_{0}+1 / 10 p=5+1 / 10 p ;$ $w=4.18$.

Les conditions (46), (47) sont claires et on voit que l'on a

$$
\begin{aligned}
& 4 \lambda_{0}= 20>19.8580 \ldots \\
&= {\left[\frac{2}{w\left(1-\frac{1}{4 t_{1} \lambda_{0}}\right)-2}+1\right] t_{0}+} \\
&+4\left[\frac{1}{w\left(1-\frac{1}{4 t_{1} \lambda_{0}}\right)-2}+\sqrt{w}+\frac{1}{2} \sqrt{\frac{t_{0}}{2 \lambda_{0}}}\right] t_{1} . \\
&-231-
\end{aligned}
$$




\section{Dong Pingping}

On en déduit

$$
4 \lambda\left(1-\frac{1}{8 \lambda_{0}^{2} p}\right)^{2} \geq 4 \lambda_{0}+\frac{0.1986}{p}>19.8580 \ldots+\frac{0.1207}{p} \geq \tau_{1} ;
$$

c'est (48).

Les conditions (49), (50) peuvent être facilement vérifiées.

Alors on a les minorations (27) dans le cas $1,(28)$ dans le cas 2, (29) dans le cas 3 et (45) dans le cas 4.

$$
\begin{gathered}
\lambda^{2} \leq 25+1.0034 \frac{1}{p} \\
125+7.5 \frac{1}{p} \leq \lambda^{3} \leq 125+7.5502 \frac{1}{p} \\
\lambda^{4} \leq 625+50.5023 \frac{1}{p} \\
\left\{\begin{array}{l}
c_{1}=\frac{1}{\log p}\left(4.75+0.095 \frac{1}{p}\right) \\
c^{2} \leq \frac{625}{\log ^{2} p}\left(25+1.0034 \frac{1}{p}\right) \leq \frac{25}{\log ^{4} p}\left(p^{2}+0.0809 p\right) \\
c_{0} \geq \frac{550}{\log ^{3} p}\left(p^{2}+0.06 p\right) \\
\chi_{1}<0.3334, \quad \chi_{2}<2.0463, \quad \chi<2.3763 \frac{1}{p}
\end{array}\right.
\end{gathered}
$$

Dans les premiers trois cas, on a même de meilleurs résultats.

Cas 1. $\left(16 \chi_{1} c \log ^{2} p\right) / p=16 \chi_{1} \lambda^{2} \leq 136$, alors par (27), on a

$$
\begin{aligned}
\log |\Lambda| & \geq-\frac{1}{8 \log ^{3} p}(5.6485+7 \log p) D a_{1} a_{2} \log B \\
& \geq-\frac{1.5177}{\log ^{2} p} D a_{1} a_{2} \log B
\end{aligned}
$$

Cas 2. $4 \chi_{2} c \leq\left(204.63 / \log ^{2} p\right)(p+0.0402)$, alors par (28)

$$
\begin{aligned}
\log |\Lambda| & \geq-\left[\frac{\log 2}{8 \log ^{3} p}+\frac{204.63}{\log ^{2} p}(p+0.0402)\right] D^{3} a_{1} a_{2} \log B \\
& \geq-207.3984 \frac{p}{\log ^{2} p} D^{3} a_{1} a_{2} \log B .
\end{aligned}
$$


Cas 3. $c_{0} / 2 c=t_{0} \lambda p / 2 \log p \leq 11.0734(p / \log p)$, alors par (29)

$$
\log |\Lambda| \geq-\left(\frac{\log 2}{4 \log ^{2} p}+\frac{c_{0}}{2 c}\right) D^{2} a_{1} a_{2} \geq-11.1260 \frac{p}{\log p} D^{2} a_{1} a_{2} .
$$

Cas 4. On a :

$$
\begin{aligned}
4\left(\frac{1}{p-1}+\frac{1}{p^{2}}\right) c^{2} \log p & \leq \frac{2500}{\log ^{3} p}\left(\frac{1}{p-1}+\frac{1}{p^{2}}\right)\left(p^{2}+0.0809 p\right) \\
& \leq \frac{2500}{\log ^{3} p}\left(\frac{1}{p-1}+\frac{1}{p^{2}}\right) p^{2}+123.5973 \frac{p}{\log ^{3} p}
\end{aligned}
$$

Par (53), (56) :

$$
\begin{gathered}
c_{5} \leq \tau_{1} \frac{\lambda^{3} p^{2}}{\log ^{3} p} \leq \frac{p^{2}}{\log ^{3} p} \lambda^{3}\left(20+\frac{0.1986}{p}\right) \leq \frac{2500}{\log ^{3} p}\left(p^{2}+0.0706 p\right) \\
2\left[\frac{1}{\log p}+\frac{\log \left(4 c \log ^{2} p\right)}{4 \log ^{2} p}\right] c \leq \frac{126.945}{\log ^{3} p}(p+0.0402) \\
4\left(\frac{1}{p-1}+\frac{1}{p^{2}}\right) c^{2} \log p+c_{5}+2\left[\frac{1}{\log p}+\frac{\log \left(4 c \log ^{2} p\right)}{4 \log ^{2} p}\right] c \leq \\
\leq \frac{2500}{\log ^{3} p}\left[\left(\frac{1}{p-1}+\frac{1}{p^{2}}\right) p^{2}+0.1715 p\right]
\end{gathered}
$$

Par (51) :

$$
\eta>\frac{2}{w\left(1-\frac{1}{4 t_{1} \lambda}\right)-2}>\eta^{\prime}=1.0204-0.0024 \frac{1}{p} .
$$

Remarquons (54) :

$$
\begin{aligned}
T_{1}= & c_{0} \log p \cdot D^{4} a_{1} a_{2} \log B+ \\
- & {\left[p\left(c+\frac{c_{1}}{2}\right)+\frac{1}{8 \log ^{2} p}\left(\log \left(4 c_{1} \log p+1\right)+\right.\right.} \\
& \left.\left.+\frac{5}{2} \log 2+\log \zeta\right)\right] D^{3}\left(a_{1}+a_{2}\right) \log B \\
\geq & \frac{\tau}{\log ^{2} p} D^{3}\left(a_{1}+a_{2}\right) \log B \geq 0
\end{aligned}
$$




\section{Dong Pingping}

$$
\begin{aligned}
&(\eta+1) c_{0} \log p \cdot D^{4} a_{1} a_{2} \log B-c_{6} D^{3}\left(a_{1}+a_{2}\right) \log B= \\
&=\eta T_{1}+c_{0} \log p \cdot D^{4} a_{1} a_{2} \log B-\left\{p \chi c+\frac{1}{8 \log ^{2} p}\left[\log \left(4 c_{1} \log p+1\right)+\right.\right. \\
&\left.\left.+\frac{3}{2} \log 2+\log \zeta\right]+\frac{1}{8 \log p}\right\} D^{3}\left(a_{1}+a_{2}\right) \log B \\
& \geq \eta^{\prime} T_{1}+c_{0} \log p \cdot D^{4} a_{1} a_{2} \log B-\left\{p \chi c+\frac{1}{8 \log ^{2} p}\left[\log \left(4 c_{1} \log p+1\right)+\right.\right. \\
&\left.\left.\quad+\frac{3}{2} \log 2+\log \zeta\right]+\frac{1}{8 \log p}\right\} D^{3}\left(a_{1}+a_{2}\right) \log B \\
&=\left(\eta^{\prime}+1\right) c_{0} \log p \cdot D^{4} a_{1} a_{2} \log B-\left\{p\left[\left(\eta^{\prime}+\chi\right) c+\eta^{\prime} \frac{c_{1}}{2}\right]+\right. \\
&+\frac{\eta^{\prime}+1}{8 \log ^{2} p}\left[\log \left(4 c_{1} \log p+1\right)+\frac{3}{2} \log 2+\log \zeta\right]+ \\
&\left.+\frac{\eta^{\prime} \log ^{2}}{8 \log ^{2} p}+\frac{1}{8 \log p}\right\} D^{3}\left(a_{1}+a_{2}\right) \log B ;
\end{aligned}
$$

en utilisant (57), (58) cette inégalité (60) est continuée en

$$
\begin{aligned}
& \geq \frac{1111.22 p^{2}}{\log ^{2} p}\left(1+0.587 \frac{1}{p}\right) D^{4} a_{1} a_{2} \log B+ \\
& -\left\{\frac{84.9175 p^{2}}{\log ^{2} p}\left[1+(0.0488+0.0001 \log \zeta) \frac{1}{p}\right]+\right. \\
& \left.\quad+\frac{2.4235 p}{\log p}\left(1+0.0697 \frac{1}{p}\right)\right\} D^{3}\left(a_{1}+a_{2}\right) \log B \\
& 2\left(\log 4+\frac{\log p}{p-1}\right) c \leq \frac{p}{\log ^{2} p} 69.315\left(1+0.7214 \frac{\log p}{p-1}\right)\left(1+0.0402 \frac{1}{p}\right) .
\end{aligned}
$$

On déduit de (45), (59), (60), (61) que

$$
\log \frac{|\Lambda|}{p} \geq-T .
$$

Supposons que $p=2$. Pour le cas 4 :

$$
\begin{aligned}
& \begin{array}{ll}
\text { les points } \alpha_{1}^{4 u} \alpha_{2}^{4 v},-U_{1} \leq u \leq U_{1}, & -V_{1} \leq v \leq V_{1} \\
& \text { sont deux à deux distincts; } \\
\text { les points } u+v \beta,-U_{2} \leq u \leq U_{2}, & -V_{2} \leq v \leq V_{2} \\
B c\left(a_{1}+a_{2}\right) \geq \frac{c_{0}}{2} D a_{1} a_{2} & \text { sont deux à deux distincts, }
\end{array}
\end{aligned}
$$


Minoration de combinaisons linéaires de deux logarithmes $p$-adiques

Pour $(u, v) \in \mathbb{Z}^{2}$, on pose

$$
\varphi(u, v)=\sum_{h=0}^{L_{0}} \sum_{k=-L_{1}}^{L_{1}} p_{h k} \Delta_{h}\left(b_{2} u+b_{1} v\right) \alpha_{1}^{4 k u} \alpha_{2}^{4 k v},
$$

où

$$
p_{h k}=\sum_{d=1}^{D_{0}} p_{h k d} \xi_{d}, \quad 0 \leq h \leq L_{0},-L_{1} \leq k \leq L_{1},
$$

$p_{h k d} \in \mathbb{Z}, \quad 0 \leq h \leq L_{0},-L_{1} \leq k \leq L_{1}, d=1,2, \ldots, D_{0}$.

Proposition 4.8. - Il existe $p_{h k d} \in \mathbb{Z}$ non tous nuls tels que

$$
\varphi(u, v)=0, \quad-M_{1} \leq u \leq M_{1},-M_{2} \leq v \leq M_{2}
$$

avec

$$
\begin{aligned}
\log \max \left\|p_{h k d}\right\| \leq & \eta\left\{c^{2} D^{3} a_{1} a_{2} \log ^{2} B+\right. \\
& \left.-2 c_{0} \log 2 \cdot D^{3} a_{1} a_{2} \log B+c_{3} D^{2}\left(a_{1}+a_{2}\right) \log B\right\}
\end{aligned}
$$

où

$$
\left\{\begin{array}{l}
c_{2}=c_{0}+8 c c_{1} \\
c_{3}=2\left(2 c+c_{1}\right)+\frac{1}{8 \log ^{2} 2}\left\{\log \left(4 c_{1}+\frac{1}{\log 2}\right)+\frac{7}{4} \log 2+1\right\} .
\end{array}\right.
$$

\section{Démonstration}

On utilise le lemme 2.1 avec " $\mu$ ", " $\nu$ " indiqués dans (23); on tire facilement de (19), (24), (25) que $\nu>D_{0} \mu$ et

$$
\begin{gathered}
D_{0} \mu /\left(\nu-D_{0} \mu\right) \leq \eta \\
\varphi(u, v)=\sum_{h=0}^{L_{0}} \sum_{k=-L_{1}}^{L_{1}} \sum_{d=1}^{D_{0}} p_{h k d} \Delta_{h}\left(b_{2} u+b_{1} v\right) \alpha_{1}^{4 k u+d_{1}} \alpha_{2}^{4 k v+d_{2}}
\end{gathered}
$$

pour $(u, v) \in \mathbb{Z}^{2}$, les coefficients de $\varphi(u, v)$ sont des polynômes en $\alpha_{1}, \alpha_{2}$, $\alpha_{1}^{-1}, \alpha_{2}^{-1}$ à coefficients entiers dont les degrés par rapport à $\alpha_{1}, \alpha_{2}, \alpha_{1}^{-1}$, $\alpha_{2}^{-1}$ sont inférieurs ou égaux à

$$
\begin{array}{ll}
N_{1}(u, v)=4 L_{1}\|u\|+D-1 & N_{2}(u, v)=4 L_{1}\|v\|+D-1 \\
N_{1}^{\prime}(u, v)=4 L_{1}\|u\| & N_{2}^{\prime}(u, v)=4 L_{1}\|v\|
\end{array}
$$

respectivement. 


\section{Dong Pingping}

Pour $(u, v) \in \mathbb{Z}^{2},-M_{1} \leq u \leq M_{1},-M_{2} \leq v \leq M_{2}$ et pour $0 \leq h \leq L_{0}$, on a grâce à (64) :

$$
\begin{aligned}
\max \left(\left\|b_{2} u+b_{1} v\right\|, \frac{h}{2}\right) & \leq \max \left(B\left(M_{1}+M_{2}\right), \frac{L_{0}}{2}\right) \\
& \leq \max \left\{B c\left(a_{1}+a_{2}\right) D^{2} \log B, \frac{c_{0}}{2} D^{3} a_{1} a_{2} \log B\right\} \\
& =E
\end{aligned}
$$

où on a toujours posé $E=b c D^{2}\left(a_{1}+a_{2}\right) \log B$, et on a

$$
\left\|\Delta_{h}\left(b_{2} u+b_{1} v\right)\right\| \leq 2 \frac{E^{h}}{h !} .
$$

La somme $L(u, v)$ des longueurs de tous les coefficients de $\varphi(u, v)$ vérifie

$$
\begin{aligned}
L(u, v) & \leq D\left(2 L_{1}+1\right) \sum_{h=0}^{L_{0}}\left\|\Delta_{h}\left(b_{2} u+b_{1} v\right)\right\| \\
& \leq 2 D\left(2 L_{1}+1\right) \sum_{h=0}^{L_{0}} \frac{E^{h}}{h !} \\
& \leq 2 D\left(2 L_{1}+1\right)\left(\frac{B}{4}\right)^{L_{0}} \sum_{h=0}^{L_{0}} \frac{1}{h !}\left(4 c D^{2}\left(a_{1}+a_{2}\right) \log B\right)^{h} \leq L
\end{aligned}
$$

avec

$$
L=2 D\left(2 L_{1}+1\right)\left(\frac{B}{4}\right)^{c_{0} D^{3} a_{1} a_{2} \log B} B^{4 c D^{2}\left(a_{1}+a_{2}\right)}
$$

donc

$$
\begin{aligned}
V(u, v) & =L(u, v) e^{N_{1}(u, v) h\left(\alpha_{1}\right)+N_{2}(u, v) h\left(\alpha_{2}\right)+N_{1}^{\prime}(u, v) h\left(\alpha_{1}^{-1}\right)+N_{2}^{\prime}(u, v) h\left(\alpha_{2}^{-1}\right)} \\
& \leq L e^{8 L_{1}\left(a_{1}\|u\|+a_{2}\|v\|\right)+(D-1)\left(a_{1}+a_{2}\right)} .
\end{aligned}
$$

Alors le lemme 2.1 dit que le système linéaire (65) possède une solution

$$
p_{h k d} \in \mathbb{Z}, \quad 0 \leq h \leq L_{0},-L_{1} \leq k \leq L_{1}, d=1,2, \ldots, D_{0}
$$

non nulle vérifiant

$\log \max \left\|p_{h k d}\right\| \leq$ 
Minoration de combinaisons linéaires de deux logarithmes p-adiques

$$
\begin{aligned}
\leq & \frac{1}{\nu-D_{0} \mu}\left\{\mu \log 2+D_{0} \sum_{u=-M_{1}}^{M_{1}} \sum_{v=-M_{2}}^{M_{2}} \log V(u, v)\right\} \\
\leq & \frac{1}{\nu-D_{0} \mu}\left\{\mu \log 2+D_{0}[\mu \log L+\right. \\
& \left.\left.+8 L_{1} \sum_{u=-M_{1}}^{M_{1}} \sum_{v=-M_{2}}^{M_{2}}\left(a_{1}\|u\|+a_{2}\|v\|\right)+\mu(D-1)\left(a_{1}+a_{2}\right)\right]\right\} \\
= & \frac{1}{\nu-D_{0} \mu}\left\{\mu \log 2+D_{0}\left[\mu \log L+8 L_{1}\left(M_{1}\left(M_{1}+1\right)\left(2 M_{2}+1\right) a_{1}+\right.\right.\right. \\
& \left.\left.\left.+M_{2}\left(M_{2}+1\right)\left(2 M_{2}+1\right) a_{2}\right)+\mu(D-1)\left(a_{1}+a_{2}\right)\right]\right\} \\
\leq & \frac{D_{0} \mu}{\nu-D_{0} \mu}\left\{\log 2+\log L+4 L_{1}\left[\left(M_{1}+\frac{1}{2}\right) a_{1}+\left(M_{2}+\frac{1}{2}\right) a_{2}\right]+\right. \\
& \left.+(D-1)\left(a_{1}+a_{2}\right)\right\} \\
\leq & \eta\left\{\log L+4 L_{1}\left(M_{1} a_{1}+M_{2} a_{2}\right)+\log 2+\left(2 L_{1}+D-1\right)\left(a_{1}+a_{2}\right)\right\} \\
& \log \log B \leq \log \left(\frac{e}{2 \log 2} \log B\right) \leq \frac{\log B}{2 \log 2}
\end{aligned}
$$

$$
\begin{aligned}
\log 2 D\left(2 L_{1}+1\right) & \leq \log \left[2\left(2 c_{1}+\frac{1}{2 \log 2}\right) D^{2} \log B\right] \\
& \leq \log \left[2\left(2 c_{1}+\frac{1}{2 \log 2}\right)\right]+\frac{\log 2}{2} D^{2}+\frac{\log B}{2 \log 2} \\
& \leq c_{4} D^{2}\left(a_{1}+a_{2}\right) \log B
\end{aligned}
$$

avec

$$
c_{4}=\frac{1}{8 \log ^{2} 2}\left[\log \left(4 c_{1}+\frac{1}{\log 2}\right)+\frac{1}{2} \log 2+1\right] .
$$

On a aussi

$$
\left\{\begin{array}{l}
L_{1}\left(M_{1} a_{1}+M_{2} a_{2}\right) \leq 2 c c_{1} D^{3} a_{1} a_{2} \log ^{2} B \\
\log 2+\left(2 L_{1}+D-1\right)\left(a_{1}+a_{2}\right) \\
\quad \leq \log 2+\left(2 c_{1} D \log B+D-1\right)\left(a_{1}+a_{2}\right) \\
\quad \leq\left(2 c_{1} \log B+\frac{D-1}{D^{2}}+\frac{1}{4 D^{2}}\right) D^{2}\left(a_{1}+a_{2}\right) \\
\quad \leq\left(2 c_{1}+\frac{5}{32 \log 2}\right) D^{2}\left(a_{1}+a_{2}\right) \log B
\end{array}\right.
$$

On en déduit (66) de (67), (68) et (69). 


\section{Dong Pingping}

On voit facilement que $p_{h k}, 0 \leq h \leq L_{0},-L_{1} \leq k \leq L_{1}$ ne sont pas tous nuls et que

$$
\left|p_{h k}\right| \leq 1, \quad 0 \leq h \leq L_{0},-L_{1} \leq k \leq L_{1} .
$$

On pose

$$
F(z)=\sum_{h=0}^{L_{0}} \sum_{k=-L_{1}}^{L_{1}} p_{h k} \Delta_{h}\left(b_{2} z\right) \alpha_{1}^{4 k z}, \quad z \in \mathbb{C}_{p}, \quad \nu(z) \geq-2
$$

de la sorte que

$$
F(u+v \beta)=\sum_{h=0}^{L_{0}} \sum_{k=-L_{1}}^{L_{1}} p_{h k} \Delta_{h}\left(b_{2} u+b_{1} v\right) \alpha_{1}^{4 k u} \alpha_{1}^{4 k v \beta},
$$

donc

$$
\varphi(u, v)-F(u+v \beta)=\sum_{h=0}^{L_{0}} \sum_{k=-L_{1}}^{L_{1}} p_{h k} \Delta_{h}\left(b_{2} u+b_{1} v\right) \alpha_{1}^{4 k u}\left(\alpha_{2}^{4 k v}-\alpha_{1}^{4 k v \beta}\right) .
$$

On utilise le lemme 2 pour obtenir :

$$
\begin{aligned}
|\varphi(u, v)-F(u, v \beta)| & \leq \max _{-L_{1} \leq k \leq L_{1}}\left|\alpha_{2}^{4 k v}-\alpha_{1}^{4 k v \beta}\right| \\
& =\max _{-L_{1} \leq k \leq L_{1}}\left|\frac{4 k v}{b_{2}}\right||\Lambda| \leq \frac{|\Lambda|}{4}
\end{aligned}
$$

pour tout $(u, v) \in \mathbb{Z}^{2}$.

Proposition 4.9. - Sous l'hypothèse (H), on a

$$
\log |\varphi(u, v)| \leq \max \left\{\log |F|_{4}-2 \mu \log 2, \log \frac{|\Lambda|}{4}-\log \Delta\right\}
$$

pour tout $(u, v) \in \mathbb{Z}^{2}$ et

$$
\begin{aligned}
\log |F|_{4}-2 \mu \log 2<- & {\left[2\left(2 c-\frac{1}{4 \log ^{2} 2}\right)^{2} D^{4} a_{1} a_{2} \log ^{2} B+\right.} \\
& \left.-3 c_{0} D^{3} a_{1} a_{2} \log B\right] \log 2 .
\end{aligned}
$$


Minoration de combinaisons linéaires de deux logarithmes p-adiques

Démonstration. - La fonction $F$ est analytique sur le disque $|z| \leq 4$ et vérifie

$$
|F|_{4} \leq \max _{0 \leq h \leq L_{0}}\left|\Delta_{h}\left(b_{2} z\right)\right|_{4}=\max _{0 \leq h \leq L_{0}}\left|\Delta_{h}(z)\right|_{4}<8^{L_{0}} .
$$

Pour $\gamma=u^{\prime}+v^{\prime} \beta \in \Gamma,\left\|u^{\prime}\right\| \leq M_{1},\left\|v^{\prime}\right\| \leq M_{2}$, on a $\varphi\left(u^{\prime}, v^{\prime}\right)=0$, donc

$$
|F(\gamma)|=\left|F(\gamma)-\varphi\left(u^{\prime}, v^{\prime}\right)\right| \leq \frac{|\Lambda|}{4} .
$$

Le lemme 2.3 avec $R=4, r=1,\left\{z_{1}, \ldots, z_{s}\right\}=\Gamma, s=|\Gamma|=\mu$ donne

$$
\begin{aligned}
|F|_{1} & \leq \max \left\{|F|_{4} 4^{-\mu}, \frac{|F(\gamma)|}{\Delta}, \gamma \in \Gamma\right\} \\
& \leq \max \left\{|F|_{4} 4^{-\mu}, \frac{|\Lambda|}{4 \Delta}\right\} .
\end{aligned}
$$

Pour $(u, v) \in \mathbb{Z}^{2}$, on a

$$
\begin{aligned}
|\varphi(u, v)| & \leq \max (|\varphi(u, v)-F(u+v \beta)|,|F(u+v \beta)|) \\
& \leq \max \left(\frac{|\Lambda|}{4},|F|_{1}\right) \leq \max \left(|F|_{4} 4^{-\mu}, \frac{|\Lambda|}{4}\right)
\end{aligned}
$$

on a donc (71) et

$$
\begin{aligned}
& \log |F|_{4}-2 \mu \log 2<L_{0} \log 8-2 \mu \log 2= \\
& \quad=-\left(2 \mu-3 L_{0}\right) \log 2 \\
& \quad \leq-\left[2\left(2 c-\frac{1}{4 \log ^{2} 2}\right)^{2} D^{4} a_{1} a_{2} \log ^{2} B-3 c_{0} D^{3} a_{1} a_{2} \log B\right] \log 2 .
\end{aligned}
$$

Proposition 4.10. - On pose $(u, v) \in \mathbb{Z}^{2},\|u\| \leq M_{1}^{*},\|v\| \leq M_{2}^{*}$. Alors ou bien $\varphi(u, v)=0$ ou bien

$$
\log |\varphi(u, v)| \geq-S_{1}
$$

où

$S_{1}=c_{5} D^{4} a_{1} a_{2} \log ^{2} B-2(\eta+1) c_{0} \log 2 \cdot D^{4} a_{1} a_{2} \log B+c_{6} D^{3}\left(a_{1}+a_{2}\right) \log B$ 


\section{Dong Pingping}

avec

$$
\begin{aligned}
c_{5}= & 2(\eta+1) c_{0}+8(\eta+2 \chi) c c_{1} \\
c_{6}= & 2 \eta\left(2 c+c_{1}\right)+\frac{\eta+1}{8 \log ^{2} 2}\left[\log \left(4 c_{1}+\frac{1}{\log 2}\right)+\frac{7}{4} \log 2+1\right]+ \\
& -\frac{1}{32 \log 2}+4 \chi c .
\end{aligned}
$$

Démonstration. - Nous utilisons le lemme 2.4 (b).

$$
\varphi(u, v)=\sum_{h=0}^{L_{0}} \sum_{k=-L_{1}}^{L_{1}} \sum_{d=1}^{D_{0}} p_{h k d} \Delta_{h}\left(b_{2} u+b_{1} v\right) \alpha_{1}^{4 k u+d_{1}} \alpha_{2}^{4 k v+d_{2}}
$$

est un polynôme en $\alpha_{1}, \alpha_{2}, \alpha_{1}^{-1}, \alpha_{2}^{-1}$ à coefficients entiers. soient $N_{1}, N_{2}$, $N_{1}^{\prime}, N_{2}^{\prime}$ les degrés de $\varphi$ par rapport à $\alpha_{1}, \alpha_{2}, \alpha_{1}^{-1}, \alpha_{2}^{-1}$ respectivement. On voit que

$$
\begin{array}{ll}
N_{1} \leq 4 L_{1} M_{1}^{*}+D-1 & N_{2} \leq 4 L_{1} M_{2}^{*}+D-1 \\
N_{1}^{\prime} \leq 4 L_{1} M_{1}^{*} & N_{2}^{\prime} \leq 4 L_{1} M_{2}^{*} .
\end{array}
$$

De plus, le nombre

$$
S=N_{1} h\left(\alpha_{1}\right)+N_{2} h\left(\alpha_{2}\right)+N_{1}^{\prime} h\left(\alpha_{1}^{-1}\right)+N_{2}^{\prime} h\left(\alpha_{2}^{-1}\right)
$$

est majoré par

$$
\begin{aligned}
& \left(N_{1}+N_{1}^{\prime}\right) a_{1}+\left(N_{2}+N_{2}^{\prime}\right) a_{2} \leq \\
& \quad \leq 8 L_{1}\left(M_{1}^{*} a_{1}+M_{2}^{*} a_{2}\right)+(D-1)\left(a_{1}+a_{2}\right) \\
& \quad \leq 16 \chi c c_{1} a_{1} a_{2} D^{3} \log ^{2} B+\frac{1}{8 \log 2} D^{2}\left(a_{1}+a_{2}\right) \log B .
\end{aligned}
$$

Pour $0 \leq h \leq L_{0}$, on a grâce à (64)

$$
\begin{aligned}
\max \left(\left\|b_{2} u+b_{1} v\right\|, \frac{h}{2}\right) & \leq \max \left(B\left(M_{1}^{*}+M_{2}^{*}\right), \frac{L_{0}}{2}\right) \\
& \leq \max \left[B c \chi\left(a_{1}+a_{2}\right) D^{2} \log B, \frac{c_{0}}{2} D^{3} a_{1} a_{2} \log B\right] \\
& =\chi E \\
\left\|\Delta_{h}\left(b_{2} u+b_{1} v\right)\right\| & \leq 2 \frac{(\chi E)^{h}}{h !} .
\end{aligned}
$$


Minoration de combinaisons linéaires de deux logarithmes $p$-adiques

Ainsi, la longueur de $\varphi$ vérifie

$$
\begin{aligned}
L(\varphi) & \leq \sum_{h=0}^{L_{0}} \sum_{k=-L_{1}}^{L_{1}} \sum_{d=1}^{D_{0}}\left\|p_{h k d}\right\|\left\|\Delta_{h}\left(b_{2} u+b_{1} v\right)\right\| \\
& \leq 2 D\left(2 L_{1}+1\right) \max \left\|p_{h k d}\right\| \sum_{h=0}^{L_{0}} \frac{(\chi E)^{h}}{h !} \\
& \leq 2 D\left(2 L_{1}+1\right) \max \left\|p_{h k d}\right\|\left(\frac{B}{4}\right)^{L_{0}} \sum_{h=0}^{L_{0}} \frac{\left(4 \chi c D^{2}\left(a_{1}+a_{2}\right) \log B\right)^{h}}{h !} \\
& \leq 2 D\left(2 L_{1}+1\right) \max \left\|p_{h k d}\right\|\left(\frac{B}{4}\right)^{c_{0} D^{3} a_{1} a_{2} \log B} B^{4 \chi c D^{2}\left(a_{1}+a_{2}\right)} .
\end{aligned}
$$

On utilise (68) et la proposition 4.8 :

$$
\begin{aligned}
& \log L(\varphi) \leq \\
& \leq c_{4} D^{2}\left(a_{1}+a_{2}\right) \log B+ \\
&+\eta\left\{c_{2} D^{3} a_{1} a_{2} \log ^{2} B-2 c_{0} \log 2 \cdot D^{3} a_{1} a_{2} \log B+\right. \\
&\left.+c_{3} D^{2}\left(a_{1}+a_{2}\right) \log B\right\}+ \\
&+c_{0} D^{3} a_{1} a_{2} \log ^{2} B-2 c_{0} \log 2 \cdot D^{3} a_{1} a_{2} \log B+ \\
&+4 \chi c D^{2}\left(a_{1}+a_{2}\right) \log B \\
& \leq\left(c_{0}+\eta c_{2}\right) D^{3} a_{1} a_{2} \log ^{2} B-2(\eta+1) c_{0} \log 2 \cdot D^{3} a_{1} a_{2} \log B+ \\
&+\left(c_{4}+\eta c_{3}+4 \chi c\right) D^{2}\left(a_{1}+a_{2}\right) \log B .
\end{aligned}
$$

Si $\varphi(u, v) \neq 0$, alors

$$
\begin{aligned}
\log \mid & \varphi(u, v) \mid \geq \\
\geq & -D(\log L(\varphi)+S) \\
\geq & -\left(c_{0}+\eta c_{2}+16 \chi c c_{1}\right) D^{4} a_{1} a_{2} \log ^{2} B+ \\
& +2(\eta+1) c_{0} \log 2 \cdot D^{4} a_{1} a_{2} \log B+ \\
& -\left(c_{4}+\eta c_{3}+4 \chi c+\frac{1}{8 \log 2}\right) D^{3}\left(a_{1}+a_{2}\right)(\log B)
\end{aligned}
$$

mais

$$
c_{5}=c_{0}+\eta c_{2}+16 \chi c c_{1}, \quad c_{6}=c_{4}+\eta c_{3}+4 \chi c+\frac{1}{8 \log 2} .
$$




\section{Dong Pingping}

Nous imposons ici deux nouvelles restrictions sur $c, c_{0}, c_{1}$ et $\chi$ :

$$
\begin{aligned}
2\left(2 c-\frac{1}{4 \log ^{2} 2}\right)^{2} \log 2 & \geq c_{5} \\
(2 \eta-1) c_{0} \log ^{2} 2 & \geq c_{6} .
\end{aligned}
$$

On pose

$$
P(X, Y)=\sum_{h=0}^{L_{0}} \sum_{k=-L_{1}}^{L_{1}} p_{h k} \Delta_{h}\left(b_{2} X\right) Y^{L_{1}+k} \in \mathbb{C}[X, Y],
$$

on déduit que $P \neq 0$ du fait que les $p_{h k}, 0 \leq h \leq L_{0},-L_{1} \leq k \leq L_{1}$ ne sont pas tous nuls.

Le polynôme $P$ est de degré au plus $L_{0}$ et $2 L_{1}$ par rapport à $X$ et $Y$ respectivement.

L'hypothèse (21) implique $U_{2} \geq U_{1}, V_{2} \geq V_{1} ;$ (63) implique que les points

$$
u+v \beta, \quad-U_{1} \leq u \leq U_{1}, \quad-V_{1} \leq v \leq V_{1}
$$

sont deux à deux distincts.

La première partie de (21) implique

$$
\begin{aligned}
\left(2 \chi_{1} c\right. & \left.-\frac{1}{4 \log ^{2} 2}\right)^{2} 2 \log 2>c_{0} \geq \frac{c_{1}}{2} \\
\left(2 U_{1}+1\right)\left(2 V_{1}+1\right) & \geq\left(2 \chi_{1} c-\frac{1}{4 \log ^{2} 2}\right)^{2} D^{4} a_{1} a_{2} \log ^{2} B \\
& \geq\left(2 \chi_{1} c-\frac{1}{4 \log ^{2} 2}\right)^{2} 2 \log 2 \cdot D^{3} a_{1} a_{2} \log B \\
& >c_{0} D^{3} a_{1} a_{2} \log B \geq L_{0} \text { et } 2 L_{1} .
\end{aligned}
$$

La deuxième partie de (21) implique

$$
\begin{gathered}
\left(2 \chi_{2} c-\frac{1}{4 \log ^{2} 2}\right)^{2}>4 c_{0} c_{1} \\
\left(2 U_{2}+1\right)\left(2 V_{2}+1\right) \geq\left(2 \chi_{2} c-\frac{1}{4 \log ^{2} 2}\right)^{2} D^{4} a_{1} a_{2} \log ^{2} B \\
>4 c_{0} c_{1} D^{4} a_{1} a_{2} \log B \geq 2 L_{0} \cdot 2 L_{1} . \\
-242-
\end{gathered}
$$


Minoration de combinaisons linéaires de deux logarithmes $p$-adiques

On voit aussi que :

$$
\begin{aligned}
M_{1}^{*} & \geq\left(\chi-\frac{1}{4 c \log ^{2} 2}\right) c D^{2} a_{2} \log B \\
& \geq\left(\chi_{1}+\chi_{2}\right) c D^{2} a_{2} \log B \geq U_{1}+U_{2} \\
M_{2}^{*} & \geq\left(\chi-\frac{1}{4 c \log ^{2} 2}\right) c D^{2} a_{1} \log B \\
& \geq\left(\chi_{1}+\chi_{2}\right) c D^{2} a_{1} \log B \geq V_{1}+V_{2} .
\end{aligned}
$$

En remarquant (62), (63), il résulte du lemme 2.6 qu'il existe un point $\left(u_{0}, v_{0}\right) \in \mathbb{Z}^{2},\left\|u_{0}\right\| \leq M_{1}^{*},\left\|v_{0}\right\| \leq M_{2}^{*}$ tel que $P\left(u_{0}+v_{0} \beta, \alpha_{1}^{4 u_{0}} \alpha_{2}^{4 v_{0}}\right) \neq 0$, ce qui équivaut à

$$
\varphi\left(u_{0}, v_{0}\right) \neq 0 .
$$

Puisque $\chi_{2} \geq 1$, on a $U_{2} \geq M_{1}, V_{2} \geq M_{2}$, (63) implique l'hypothèse (H). Alors, on utilise les propositions 4.9 et 4.10 , avec $(u, v)=\left(u_{0}, v_{0}\right)$ :

$$
\max \left\{\log |F|_{4}-2 \mu \log 2, \log \frac{|\Lambda|}{4}-\log \Delta\right\} \geq \log \left|\varphi\left(u_{0}, v_{0}\right)\right| \geq-S_{1} ;
$$

mais (74) implique

$$
3 c_{0} \log 2 \leq 2(\eta+1) c_{0} \log 2-\frac{c_{6}}{\log 2} .
$$

Alors par (72), (73) et (75), on a

$$
\begin{aligned}
\log |F|_{4}-2 \mu \log 2 \leq \\
\leq-\left\{2\left(2 c-\frac{1}{4 \log ^{2} 2}\right)^{2} D^{4} a_{1} a_{2} \log ^{2} B-3 c_{0} D^{3} a_{1} a_{2} \log B\right\} \log 2 \\
\leq-\left\{c_{5} D^{4} a_{1} a_{2} \log ^{2} B-\left[2(\eta+1) c_{0} \log 2-\frac{c_{6}}{\log 2}\right] D^{4} a_{1} a_{2} \log B\right\} \\
\leq-S_{1},
\end{aligned}
$$

donc :

$$
\log \frac{|\Lambda|}{4}-\log \Delta \geq-S_{1}
$$

$$
\begin{aligned}
\log \frac{|\Lambda|}{4} \geq & \log \Delta-S_{1} \\
\geq- & \left\{5 c^{2} \log 2+c_{5}+\frac{\log \left(64 c \log ^{2} 2\right)}{2 \log ^{2} 2} c\right\} D^{4} a_{1} a_{2} \log ^{2} B+ \\
& +2(\eta+1) c_{0} \log 2 \cdot D^{4} a_{1} a_{2} \log B+ \\
& -\left(c_{6}+6 \log 2 \cdot c\right) D^{3}\left(a_{1}+a_{2}\right) \log B \\
- & 243-
\end{aligned}
$$




\section{Dong Pingping}

Maintenant nous nous mettons à choisir $c, c_{0}, c_{1}$ et puis $\chi_{1}, \chi_{2}, \chi$.

Soient $t_{0}, t_{1}, \lambda_{0}, \lambda$ des constantes absolues satisfaisant les conditions suivantes :

$$
\begin{aligned}
& \frac{2}{3} \leq t_{1}, \quad 3 t_{1} \leq t_{0}, \quad \lambda>\lambda_{0} \geq 2, \\
& w=t_{0} t_{1} \geq 3,
\end{aligned}
$$

et

$$
\begin{aligned}
8 \lambda\left(1-\frac{1}{16 \lambda^{2}}\right)^{2} \geq \tau_{1} & =\left(\frac{2}{w Z_{0}-2}+1\right) t_{0}+ \\
& -8\left[\frac{1}{w Z_{0}-2}+\sqrt{w}+\frac{1}{2} \sqrt{\frac{t_{0}}{2 \lambda}}+\frac{1}{4 \lambda_{0}^{2}}\right] t_{1},
\end{aligned}
$$

où $Z_{0}=\left(1-\left(1 / 4 t_{1} \lambda\right)\right)\left(1+\left(1 / 16 \lambda^{2}\right)\right)^{-2}$,

$$
\begin{aligned}
& \left(\frac{4}{w Z_{0}-2}-1\right) 4 t_{0} \lambda^{3} \geq \\
& \geq \frac{4}{w Z_{0}-2}\left(\frac{4 \lambda^{2}}{\log 2}+t_{1} \lambda\right)+ \\
& \quad+\frac{1}{8 \log 2}\left(\frac{2}{w Z_{0}-2}+1\right)\left(\log \frac{4 t_{1} \lambda+1}{\log 2}+\frac{7}{4} \log 2+1\right)-\frac{1}{32} .
\end{aligned}
$$

Choisissons

$$
c_{1}=\frac{t_{1} \lambda}{\log 2}, \quad c=\frac{2 \lambda^{2}}{\log ^{2} 2}, \quad c_{0}=\frac{4 t_{0} \lambda^{3}}{\log ^{3} 2} .
$$

(77) signifie que $c \geq 2, c_{0} \geq 1, c_{1} \geq 1 / \log 2$ et $2 \log ^{2} 2 \cdot c_{0} \geq c_{1}$ et on déduit aussi que $t_{1} \geq 2 / 3, \lambda>2$ et (78) que

$$
\begin{aligned}
Z & =c_{0}\left(2 c_{1}-\frac{1}{2 \log 2}\right)\left(2 c+\frac{1}{4 \log ^{2} 2}\right)^{-2} \\
& =\frac{c_{0} c_{1}}{2 c_{2}}\left(1-\frac{1}{4 c_{1} \log 2}\right)\left(1+\frac{1}{8 c \log ^{2} 2}\right)^{-2} \\
& =\frac{w}{2} Z_{0}>1 ;
\end{aligned}
$$

alors (19), (20) sont vérifiées. On voit que

$$
\begin{gathered}
\eta=\frac{1}{Z-1}=\frac{2}{w Z_{0}-2} . \\
-244-
\end{gathered}
$$


Choisissons de plus

$$
\begin{gathered}
\chi_{1}=\frac{1}{2} \sqrt{\frac{t_{0}}{2 \lambda}}+\frac{1}{16 \lambda_{0}^{2}}, \quad \chi_{2}=\sqrt{w}+\frac{1}{16 \lambda_{0}^{2}} \\
\chi=\sqrt{w}+\frac{1}{2} \sqrt{\frac{t_{0}}{2 \lambda}}+\frac{1}{4 \lambda_{0}^{2}} .
\end{gathered}
$$

En utilisant (77), (78), les relations (21), (22) deviennent claires.

On utilise (81), (82) et puis (79) :

$$
\begin{aligned}
2\left(2 c-\frac{1}{4 \log ^{2} 2}\right)^{2} \log 2 & =\frac{32 \lambda^{4}}{\log ^{3} 2}\left(1-\frac{1}{16 \lambda^{2}}\right)^{2} \\
& \geq \tau_{1} \frac{4 \lambda^{3}}{\log ^{3} 2}=c_{5}
\end{aligned}
$$

c'est (73). On divise (80) par $\log 2$, on obtient (74).

Choisissons $t_{0}=4.65, t_{1}=0.9, \lambda_{0}=3.9254, \lambda=3.9255, w=4.185$.

Les conditions (77), (78) sont claires, et on voit que l'on a

$$
8 \lambda\left(1-\frac{1}{16 \lambda^{2}}\right)^{2}=31.1497 \ldots>31.1494 \ldots=\tau_{1}
$$

c'est (79).

On voit par les calculs que la condition (80) est largement satisfaite.

Alors, on a les minorations (27) dans le cas 1, (28) dans le cas 2, (29) dans le cas 3 , et (76) dans le cas 4 .

$$
\begin{aligned}
& \lambda^{2}=15.4095 \ldots, \quad \lambda^{3}=60.4901 \ldots, \quad \lambda^{4}=237.4542 \ldots \\
& \quad\left\{\begin{array}{l}
c_{1} \leq 5.0974, \quad c \leq 64.3497, \quad c^{2}=\frac{949.8170}{\log ^{4} 2} \\
c_{0} \geq \frac{2341.4275}{\log 2}
\end{array}\right. \\
& \chi_{1}=0.3888 \ldots, \quad \chi_{2}=2.0497 \ldots, \quad \chi=2.4467 \ldots
\end{aligned}
$$

Dans les premiers trois cas, on a même de meilleurs résultats. 


\section{Dong Pingping}

Cas 1. $\chi_{1} c \leq 25.0256, \log \chi_{1} c \leq 3.2203$, alors par (27), on a

$$
\begin{aligned}
\log |\Lambda| & \geq \frac{1}{8 \log ^{3} 2}\left[\log \left(\chi_{1} c\right)+\frac{2}{e}+1+8 \log 2\right] D a_{1} a_{2} \log B \\
& \geq-3.7604 D a_{1} a_{2} \log B .
\end{aligned}
$$

Cas 2. $\chi_{2} c \leq 131.9034$, alors par (28), on a

$$
\begin{aligned}
\log |\Lambda| & \geq-\left(\frac{1}{8 \log ^{2} 2}+4 \chi_{2} c\right) D^{3} a_{1} a_{2} \log B \\
& \geq-527.8737 D^{3} a_{1} a_{2} \log B .
\end{aligned}
$$

Cas 3. $\left(c_{0} / 2 c\right)=\left(t_{0} \lambda / \log 2\right) \geq 18.2536 / \log 2$, alors par $(29)$, on a

$$
\begin{aligned}
\log |\Lambda| & \geq-\left(\frac{1}{4 \log ^{2} 2}+\frac{c_{0}}{2 c}\right) D^{2} a_{1} a_{2} \\
& \geq-26.6969 D^{2} a_{1} a_{2} .
\end{aligned}
$$

Cas 4. $5 c^{2} \log 2 \leq 4749.0850 / \log ^{3} 2$, par (83), on a

$$
\begin{aligned}
& c_{5}=\tau_{1} \frac{4 \lambda^{3}}{\log ^{3} 2} \leq \frac{7536.9330}{\log ^{3} 2} \\
& \frac{\log \left(64 c \log ^{2} 2\right)}{2 \log ^{2} 2} c=\frac{\lambda^{2} \log \left(128 \lambda^{2}\right)}{\log ^{4} 2} \leq \frac{168.6741}{\log ^{3} 2} \\
& 5 c^{2} \log 2+c_{5}+\frac{\log \left(64 c \log ^{2} 2\right)}{2 \log ^{2} 2} c \leq \frac{12454.6921}{\log ^{3} 2} \leq 37390.252 .
\end{aligned}
$$

On calcule par (81)

$$
\eta=1.0767 \ldots
$$

alors par (84)

$$
2(\eta+1) c_{0} \log 2 \geq 9724.9496 .
$$

On calcule $c_{6}$ par (84), (85) et (86) :

$$
c_{6} \leq 920.7369, \quad c_{6}+6 c \log 2 \leq 1188.3801 .
$$

Enfin, il résulte de (76) que

$$
\begin{gathered}
\log \frac{|\Lambda|}{4} \geq-T . \\
-246-
\end{gathered}
$$


Démonstration du théorème 4.1. - On considère seulement le cas $p \geq 3$ (dans le cas $p=2$ la démonstration se fait de manière semblable).

Soit $m=\left(b_{1}, b_{2}\right)>0, b_{1}=m b_{1}^{\prime}, b_{2}=m b_{2}^{\prime}$ avec deux entiers $b_{1}^{\prime}, b_{2}^{\prime}$, $\left(b_{2}^{\prime}, b_{2}^{\prime}\right)=1$. On pose

$$
\Lambda^{\prime}=\alpha_{1}^{b_{1}^{\prime}}-\alpha_{2}^{b_{2}^{\prime}} \neq 0
$$

alors, on a le lemme 2.5

$$
|\Lambda|=|m|\left|\Lambda^{\prime}\right| \geq \frac{\left|\Lambda^{\prime}\right|}{m} .
$$

Cas 1. $\max \left(\left\|b_{1}^{\prime}\right\|,\left\|b_{2}^{\prime}\right\|\right) \geq 2$. On utilise le lemme 2.4 (b) pour $\Lambda^{\prime} \neq 0$ :

$$
\begin{aligned}
\log \left|\Lambda^{\prime}\right| & \geq-D\left[\log 2+\left\|b_{1}^{\prime}\right\| h\left(\alpha_{1}\right)+\left\|b_{2}^{\prime}\right\| h\left(\alpha_{2}\right)\right] \\
& \geq-D\left[\log 2+2\left(a_{1}+a_{2}\right)\right] ;
\end{aligned}
$$

on remarque $\log p \leq(1 / 4)\left(a_{1}+a_{2}\right)$, alors par (87) :

$$
\begin{aligned}
\log \frac{|\Lambda|}{p} & \geq \log \left|\Lambda^{\prime}\right|-\log m-\log p \\
& \geq \log \left|\Lambda^{\prime}\right|-\log B-\log \frac{1}{4}\left(a_{1}+a_{2}\right) \\
& \geq-D\left[\log 2+\frac{9}{4}\left(a_{1}+a_{2}\right)\right]-\log B \geq-T
\end{aligned}
$$

Cas 2. $\max \left(\left\|b_{1}^{\prime}\right\|,\left\|b_{2}^{\prime}\right\|\right) \geq 3$. On pose $B^{\prime}=\max \left(\left\|b_{1}^{\prime}\right\|,\left\|b_{2}^{\prime}\right\|\right)$; on a $B=m B^{\prime}$ :

$$
\begin{aligned}
& \frac{2500}{\log ^{3} p}\left[\left(\frac{p}{p-1}+\frac{1}{p^{2}}\right) p^{2}+0.1715 p\right] D^{4} a_{1} a_{2} \log ^{2} B^{\prime}+ \\
&- \frac{1111.22}{\log ^{2} p}\left(1+0.0587 \frac{1}{p}\right) D^{4} a_{1} a_{2} \log B^{\prime}+\log m \leq \\
& \leq\left\{\frac{2500}{\log ^{3} p}\left[\left(\frac{p}{p-1}+\frac{1}{p^{2}}\right) p^{2}+0.1715 p\right] \log B^{\prime}+\frac{1}{4 \log ^{2} p} \log m+\right. \\
&\left.-\frac{1111.22}{\log ^{2} p}\left(1+0.0587 \frac{1}{p}\right)\right\} D^{4} a_{1} a_{2} \log B^{\prime} \\
& \leq\left\{\frac{2500}{\log ^{3} p}\left[\left(\frac{p}{p-1}+\frac{1}{p^{2}}\right) p^{2}+0.1715 p\right] \log B+\right. \\
&\left.-\frac{1111.22}{\log ^{2} p}\left(1+0.0587 \frac{1}{p}\right)\right\} D^{4} a_{1} a_{2} \log B \\
&= \frac{2500}{\log ^{3} p}\left[\left(\frac{p}{p-1}+\frac{1}{p^{2}}\right) p^{2}+0.1715 p\right] D^{4} a_{1} a_{2} \log ^{2} B+ \\
&-\frac{1111.22}{\log ^{2} p}\left(1+0.0587 \frac{1}{p}\right) D^{4} a_{1} a_{2} \log B .
\end{aligned}
$$




\section{Dong Pingping}

On pose

$$
\begin{aligned}
T^{\prime}= & \frac{2500}{\log ^{3} p}\left[\left(\frac{p}{p-1}+\frac{1}{p^{2}}\right) p^{2}+0.1715 p\right] D^{4} a_{1} a_{2} \log ^{2} B^{\prime}+ \\
& -\frac{1111.22}{\log ^{2} p}\left(1+0.0587 \frac{1}{p}\right) D^{4} a_{1} a_{2} \log B^{\prime}+ \\
& +\left\{\frac{84.9175}{\log ^{2} p} p^{2}\left[1+(0.0488+0.0001 \log \zeta) \frac{1}{p}\right]+\right. \\
& +\frac{2.4235 p}{\log p}\left(1+\frac{0.06997}{p}\right)+ \\
& \left.+\frac{69.315 p}{\log ^{2} p}\left(1+0.7214 \frac{\log p}{p-1}\right)\left(1+\frac{0.0402}{p}\right)\right\} D^{3}\left(a_{1}+a_{2}\right) \log B^{\prime} .
\end{aligned}
$$

On a donc $T^{\prime}+\log m \leq T$.

Puisque $\left(b_{1}^{\prime}, b_{2}^{\prime}\right)=1, \Lambda^{\prime} \neq 0$, la proposition 4.2 donne

$$
\log \frac{\left|\Lambda^{\prime}\right|}{p} \geq-T^{\prime}
$$

et puis

$$
\log \frac{|\Lambda|}{p} \geq \log \frac{\left|\Lambda^{\prime}\right|}{p}-\log m \geq-T^{\prime}-\log m \geq-T
$$

Démonstration du corollaire 1.1. - On considère le cas $p \geq 3$ (dans le cas $p=2$, la démonstration se fait de manière semblable) :

$$
\begin{aligned}
T \leq\{ & \frac{2500}{\log ^{3} p}\left[\left(\frac{p}{p-1}+\frac{1}{p^{2}}\right) p^{2}+0.1715 p\right] D^{4} a_{1} a_{2} \log ^{2} B+ \\
& +\left[\frac{84.9175}{\log ^{2} p} p^{2}\left(1+\frac{0.0489}{p}\right)+\frac{2.4235}{\log p} p\left(1+\frac{0.0697}{p}\right)+\right. \\
& \left.\left.+\frac{69.315}{\log ^{2} p} p\left(1+0.7214 \frac{\log p}{p-1}\right)\left(1+\frac{0.0402}{p}\right)\right]\right\} D^{3}\left(a_{1}+a_{2}\right) \log B .
\end{aligned}
$$

Grâce au lemme 4.3, on peut suposer $\log B \geq 2 \log p$, alors

$$
\begin{aligned}
& T \leq\{ \frac{2500}{\log ^{3} p}\left[\left(\frac{p}{p-1}+\frac{1}{p^{2}}\right) p^{2}+0.1715 p\right]+ \\
&+\frac{1}{2 \log ^{2} p}\left[\frac{84.9175}{\log ^{2} p} p^{2}\left(1+\frac{0.0489}{p}\right)+\frac{2.4235}{\log p} p\left(1+\frac{0.0697}{p}\right)+\right. \\
&-248-
\end{aligned}
$$


Minoration de combinaisons linéaires de deux logarithmes p-adiques

$$
\begin{aligned}
& \left.\left.+\frac{69.315}{\log ^{2} p} p\left(1+0.7214 \frac{\log p}{p-1}\right)\left(1+\frac{0.0402}{p}\right)\right]\right\} D^{4} a_{1} a_{2} \log B \\
= & \frac{1}{\log ^{3} p}\left\{2500\left[\left(\frac{p}{p-1}+\frac{1}{p^{2}}\right) p^{2}+0.1715 p\right]+\right. \\
& +\frac{1}{2}\left[\frac{84.9175}{\log p} p^{2}\left(1+\frac{0.0489}{p}\right)+2.4235 p\left(1+\frac{0.0697}{p}\right)+\right. \\
& \left.\left.+\frac{69.315}{\log p} p\left(1+0.7214 \frac{\log p}{p-1}\right)\left(1+\frac{0.0402}{p}\right)\right]\right\} D^{4} a_{1} a_{2} \log ^{2} B \\
\leq & \frac{1}{\log ^{3} p}\left[2538.6481 p^{2}+6713.3990 p+2501.8555\right] D^{4} a_{1} a_{2} \log ^{2} B \\
\leq & 2538.6481(p+1.3223)^{2} \frac{1}{\log ^{3} p} D^{4} a_{1} a_{2} \log ^{2} B
\end{aligned}
$$

la conclusion s'obtient du théorème 4.1 口

\section{Remerciements}

Je remercie mon directeur, Michel Waldschmidt, qui m'a donné beaucoup de conseils précieux pour achever cet article.

\section{Références}

[1] Gramain (F.) et Mignotte (M.) - - Fonctions entières arithmétiques, in Approximations diophantiennes et nombres transcendants, Birkhaüser, Progress in Math. 31 (1983) pp. 99-124.

[2] Malher (K.) .- Über transzendente P-adische Zahlen, Compositio Math. 2 (1935) pp. 259-275.

[3] Mignotte (M.) et Waldschmidt (M.). - Linear forms in two logarithms and Schneider's method,

(I), Math. Ann. 231 (1978) pp. 241-267; (II) Acta Arithm. 53 (1989) pp. 251-287; (III) Ann. Fasc. Sci. Toulouse 97 (1989) pp. 43-75.

[4] VAN DER POORTEN (A.J.) .- On Baker's inequality for linear forms in logarithms, Math. Proc. Camb. Phil. Soc. 80 (1976) pp. 233-248.

[5] VAN DER Poorten (A.J.) - - Linear forms in logarithms in the p-adic case, Transcendence Theory : Advances and Applications; Academic Press 1977, chap. 2, pp. 29-57. 


\section{Dong Pingping}

[6] Schinzel (A.).- On two theorems of Gel'fond and some of their applications, Acta Arithm. 13 (1967) pp. 177-236.

[7] Serre (J.P.) . - Dépendance d'exponentielles p-adiques, Sém. Delange-Pisot-Poitou, 7ème année, 1965/66, exposé 15.

[8] Shorey (T.N.), Van der Poorten (A.J.), Tijdeman (R) and Schinzed (A.) .- Applications of the Gel'fond-Baker method to diophantine equations,

Transcendence Theory : Advances and Applications; Academic Press, 1977, chap. 3, pp. 59-77.

[9] KUNRUI ( $\mathrm{YU}$ ) . - Linear forms in p-adic logarithms, Acta. Arith. 53 (1989) pp. 107-186.

[10] KUNRUI ( $\mathrm{YU}$ ) . - Linear forms in p-adic logarithms II, Compositio Math. 74 (1990) pp. 15-113. 